\title{
Poincaré inequalities for Sobolev spaces with matrix valued weights and applications to degenerate partial differential equations
}

\author{
Dario D. Monticelli ${ }^{*}$, Kevin R. Payne ${ }^{\ddagger}$ and Fabio Punzo ${ }^{\S}$
}

December 26, 2016

\begin{abstract}
For bounded domains $\Omega$, we prove that the $L^{p}$-norm of a regular function with compact support is controlled by weighted $L^{p}$-norms of its gradient, where the weight belongs to a class of symmetric non-negative definite matrix valued functions. The class of weights is defined by regularity assumptions and structural conditions on the degeneracy set where the determinant vanishes. In particular, the weight $A$ is assumed to have rank at least one when restricted to the normal bundle of the degeneracy set $\mathcal{S}$. This generalization of the classical Poincare inequality is then applied to develop a robust theory of first order $L^{p}$-based Sobolev spaces with matrix valued weight $A$. The Poincaré inequality and these Sobolev spaces are then applied to produce various results on existence, uniqueness and qualitative properties of weak solutions to boundary value problems for degenerate elliptic, degenerate parabolic and degenerate hyperbolic PDEs of second order written in divergence form, where $A$ is calibrated to the matrix of coefficients of the second order spatial derivatives. The notion of weak solution is variational in which the spatial states belong to the matrix weighted Sobolev spaces with $p=2$. For the degenerate elliptic PDEs, the Dirichlet problem is treated by the use of the Poincarè inequality and Lax-Milgram theorem, while the treatment of Cauchy-Dirichet problem for the degenerate evolution equations relies only on the Poincarè inequality and the parabolic and hyperbolic counterparts of the Lax-Milgram theorem.
\end{abstract}

Keywords: Degenerate elliptic, parabolic and hyperbolic equations, Poincarè inequalities, Sobolev spaces, matrix valued weights, Lax-Milgram theorem .

AMS subject classification: 26D10, 35A23, 35B50, 35D30, 35J25, 35J70, 35K20, $35 \mathrm{~L} 20,35 \mathrm{~L} 80,46 \mathrm{E} 35$.

*The authors are partially supported the Gruppo Nazionale per l'Analisi Matematica, la Probabilità e le loro Applicazioni (GNAMPA) of the Istituto Nazionale di Alta Matematica (INdAM)

${ }^{\dagger}$ Dipartimento di Matematica, Politecnico di Milano, Via Bonardi 9, 20133 Milano, Italy (dario.monticelli@polimi.it).

‡Dipartimento di Matematica "F. Enriques", Università degli Studi di Milano, via C. Saldini 50, 20133 Milano, Italy (kevin.payne@unimi.it).

${ }^{\S}$ Dipartimento di Matematica e Informatica, Università della Calabria, via Pietro Bucci, 87036 Arcavacata di Rende (CS), Italy (fabio.punzo@unical.it). 


\section{Introduction}

The classical Poincaré inequality ${ }^{1}$ on a bounded domain $\Omega \subset \mathbb{R}^{n}$ can be stated as: given $p \in[1, \infty)$ there exists a constant $C_{\mathcal{P}}=C_{\mathcal{P}}(\Omega, p)>0$ such that

$$
\int_{\Omega}|u|^{p} d x \leq C_{\mathcal{P}}^{p} \int_{\Omega}|\nabla u|^{p} d x, \quad u \in C_{0}^{1}(\Omega)
$$

where $C_{0}^{1}(\Omega)$ is the space of $C^{1}$ functions with compact support in $\Omega$. This simple inequality is well known to yield an impressive amount of consequences in the theory of Sobolev spaces and their applications for well-posedness results of the Dirichlet problem for elliptic equations as well as the Cauchy-Dirichlet problem for parabolic and hyperbolic equations. Particularly striking is its success in the Hilbert space setting (with $p=2$ ) and for linear equations of second order. Making use of only (1.1) plus the Lax-Milgram theorem in the elliptic case, and of its parabolic/hyperbolic variants due to J.L. Lions (by way of a Galerkin approximation), one is able to say quite a bit about well posedness for weak solutions (the so-called variational methods).

Motivated by potential applications to second order partial differential equations with degeneracies, we will generalize the inequality (1.1) by allowing for a large class of symmetric non-negative matrix valued weights $A=A(x)$; that is, for suitable $A$ we will prove that

$$
\int_{\Omega}|u|^{p} d x \leq C_{\mathcal{P}}^{p} \int_{\Omega}\langle A(x) \nabla u, \nabla u\rangle^{p / 2} d x, \quad u \in C_{0}^{1}(\Omega),
$$

where $\langle\cdot, \cdot\rangle$ is the Euclidean scalar product on $\mathbb{R}^{n}$. The assumptions on $A$ will be grouped into two sets of hypotheses (see (2.1) and (2.3)). The first set of hypotheses concerns the regularity of the function $A$ which takes values in the symmetric nonnegative matrices $\operatorname{Sym}_{n}^{+}(\mathbb{R})$ and the second set of hypotheses gives prescriptions on where and how $A$ degenerates. In particular, the degeneracy set $\mathcal{S}$ (where the determinant of $A$ vanishes) will be taken to be a finite union of compact connected $C^{2}$ submanifolds without boundary and codimension at least two, and $A$ restricted to the normal bundle $N \mathcal{S}$ of $\mathcal{S}$ must have rank at least one. Our proof of (1.2) relies on the existence of a suitable pair $w, \widetilde{w}$ of strict subsolutions to the equation $\operatorname{div}\{A(x) \nabla w\}=0$ (one near $\mathcal{S}$ and one away from $\mathcal{S}$ ). In general, the hypothesis on the rank of $A$ restricted to $N \mathcal{S}$ cannot be dropped, while the condition on the codimension of $\mathcal{S}$ is not necessary (see the examples in section 6 and Remark 3.5).

We will then put (1.2) to work, along the classical lines indicated above, to treat the Dirichlet problem and the Cauchy-Dirichlet problem for degenerate elliptic, parabolic and hyperbolic linear equations of second order. Not surprisingly, in the degenerate elliptic case, the differential operators $L$ will be in divergence form with $A(x)$ playing the role of the (degenerate) diffusion matrix; that is, we will consider equations of the form

$$
L u:=\operatorname{div}\{A(x) \nabla u\}+\langle b(x), \nabla u\rangle+c(x) u=f(x) .
$$

Results on existence and uniqueness of weak (variational) solutions to the Dirichlet problem on bounded domains $\Omega \subset \mathbb{R}^{n}$ will be given as well as weak comparison and maximum principles. For the evolution equations, we will allow for time dependence in the coefficients and in the source for the degenerate parabolic equation

$$
\partial_{t} u=\operatorname{div}\{M(x, t) \nabla u\}+\langle b(x, t), \nabla u\rangle+c(x, t) u+f(x, t)
$$

and for the degenerate hyperbolic equation

$$
\partial_{t}^{2} u=\operatorname{div}\{M(x, t) \nabla u\}+\langle b(x, t), \nabla u\rangle+c(x, t) u+f(x, t) .
$$

\footnotetext{
${ }^{1}$ Also known as the Poincaré - Sobolev inequality or the Friedrich's inequality in the case $p=2$
} 
Here the matrix valued function $M$ will be controlled by an admissible degenerate diffusion matrix on a prescribed time interval $(0, T)$; that is, we will assume that there exists an $A$ (belonging to our class) and positive constants $\mu_{1}, \mu_{2}$ such that

$$
\mu_{1} A(x) \leq M(x, t) \leq \mu_{2} A(x), \quad x \in \Omega, t \in(0, T)
$$

in the sense of quadratic forms. We will establish results on existence and uniqueness of weak solutions to the natural Cauchy-Dirichlet problems for the equations (1.4) and (1.5) in cylindrical domains $Q_{T}:=\Omega \times(0, T]$ with $T>0$. Moreover, we will prove weak comparison and maximum principles in the parabolic case. In all cases, suitable hypotheses will need to be placed on the lower order coefficients $b$ and $c$ and on the source term $f$. In particular, we will need uniform pointwise bounds on $\left|[\sqrt{A}]^{-1} b\right|,\left|[\sqrt{M}]^{-1} b\right|$ and hence the drift coefficients (if present) must tame the singularity of $[\sqrt{A}]^{-1},[\sqrt{M}]^{-1}$ on $\mathcal{S}$.

The notion of weak solutions will be the natural one in which the standard Sobolev space $H_{0}^{1}(\Omega)=W_{0}^{1,2}(\Omega)$ of spatial states used in the non-degenerate situations will be replaced by a suitably weighted version $H_{0}^{1}(\Omega ; A)=W_{0}^{1,2}(\Omega ; A)$, which is calibrated to the degenerate diffusion matrix $A$. More precisely, for $p \in[1, \infty)$ we will define $W_{0}^{1, p}(\Omega ; A)$ as the completion of $C_{0}^{1}(\Omega)$ with respect to the obvious norm

$$
\|u\|_{W^{1, p}(\Omega ; A)}:=\left[\int_{\Omega}|u|^{p} d x+\int_{\Omega}\langle A(x) \nabla u, \nabla u\rangle^{p / 2} d x\right]^{1 / p},
$$

and the space $W^{1, p}(\Omega ; A)$ as the completion with respect to $(1.7)$ of the subspace of $C^{1}(\Omega)$ with finite norm. Of course, the Poincaré inequality (1.2) passes to the completion and yields the obvious equivalent norm on $W_{0}^{1, p}(\Omega ; A)$. In preparation for the PDE applications, various elementary properties of these spaces and an effective calculus will be developed in section 4. Of particular importance will be the question of whether these spaces defined by strong derivatives can be captured in terms of a suitable notion of weak derivatives and a key role will be played by the weak quasi-gradient. This notion was introduced in [20] to address this question for a different class of matrix valued weights which yield the Poincaré inequality. In [20] the degeneracy set $\mathcal{S}$ has codimension at least 1 and is calibrated to a class of degenerate elliptic operators having a direction of uniform ellipticity, while in the present work, the degeneracy set has codimension at least 2 but a direction of uniform ellipticity need not be present (see Example 6.1). Moreover, for current and future use, we will give a unified treatment of such Sobolev spaces with matrix valued weights by assuming only that $A \in C^{0}\left(\bar{\Omega}, \operatorname{Sym}_{n}^{+}(\mathbb{R})\right)$ having degeneracy set $\mathcal{S}$ of measure zero for which the Poincaré inequality holds.

In order to place the present work in context, some additional remarks and comparisons with the vast existing literature on Poincaré inequalities, Sobolev spaces with weights and degenerate PDEs are in order. We begin with our principal motivation which is the treatment of degenerate PDEs by focusing attention directly on the singular set $\mathcal{S}$ and the properties that a degenerate diffusion matrix $A$ must satisfy in order to employ various techniques. Here, we restrict our attention to the validity of the Poincaré inequality and its implications for linear PDE problems with Dirichlet and Cauchy-Dirichlet conditions. The key structural property that emerges is the role of a degenerate diffusion matrix $A$ with thin degeneracy set $\mathcal{S}$ for which the rank of $A$ restricted to the normal bundle of $\mathcal{S}$ is at least one. This is the so-called orthogonal rank of $A$ on $\mathcal{S}$ and plays an important role in questions of nonattainability of $\mathcal{S}$ in the associated diffusion process (see Chapter 11 of [14]). This notion of orthogonal rank also plays a key role in characterizing well-posedness of distributional solutions for degenerate elliptic and parabolic equations when the singular set $\mathcal{S}$ is a thin subset of the boundary, as done in [26]. More precisely, the main question is whether one can obtain uniqueness without prescribing Dirichlet data everywhere on $\mathcal{S}$, and this occurs when such portions are not attainable for the associated diffusion process. That boundary conditions need not be placed everywhere in degenerate contexts (operators with non negative characteristic form) is well 
known from the work of Fichera [9] (see also Fichera [10] and Oleinik and Radkevič [25] and the references therein). One can view the present paper as well as [20] and [26] as continuations of the point of view of focusing attention on $\mathcal{S}$ and $A$ for equations of nonnegative characteristic form. There are various similarities, but there are key differences in these related papers. While [26] treats boundary degeneracy and [20] treats cases where $\mathcal{S}$ is partially contained in $\Omega$ but can reach the boundary, the results of the present paper will be stated for singular sets $\mathcal{S}$ which are suitably regular and contained in the interior of $\Omega$. However, boundary degeneration for $\Omega$ can be also treated here if the required hypotheses hold on a larger domain $\widetilde{\Omega}$ which contains the closure of $\Omega$ (see Corollary 3.2). While the Poincaré inequality plays a role also in [20], it does not in [26]. On the other hand, a common feature of all of these works are the construction of suitable subsolutions. In [26] such subsolutions tend to $-\infty$ as the distance from the degeneracy set goes to 0 , while here and in [20] the relevant subsolutions will remain bounded. Moreover, in [26] the distance function to $\mathcal{S}$ plays a prominent role, while here local defining functions for $\mathcal{S}$ are the key auxiliary objects.

Having discussed our point of view, we should make some comparison with the vast literature on Poincaré inequalities, Sobolev spaces and degenerate PDEs. First, for approaches involving matrix valued weights, one must note the very general and abstract program begun by Sawyer and Wheeden in their work on Hölder continuity for subelliptic linear equations [30] (and refined in [31]), which has led to the development of elegant techniques to treat local properties of weak solutions to degenerate elliptic PDEs with rough coefficients. Through their work and that of their disciples and collaborators, the Sawyer-Wheeden program has been subsequently freed from the Hilbert space setting of $L^{2}$-based spaces (and hence linear equations) to yield results on local boundedness in [22], Harnack inequalities in [23] and compact embeddings in Lebesgue spaces [4], amongst others. In addition, global results on weak existence for linear degenerate elliptic equations have been obtained for the Dirichlet problem in [27] and for the Neumann problem as well in [21], which also contains some elements of spectral theory. In all of these works, the approach is axiomatic in nature, where in order to deduce their results the authors assume a priori the validity of suitable local Poincare and Sobolev inequalities (with a strict gain in summability) adapted to the nonnegative matrix of weights $A$, as well as the existence of suitable families of Lipschitz cutoff functions. Hence the inequality (1.2), which is the focus of the present work, can be seen as a precursor of the conditions required in the Sawyer-Wheeden axiomatic program. While there may be some cases in which results of the present work overlap with results that have been obtained within the axiomatic program, neither approach subsumes the other. On the one hand, we make stronger explicit hypotheses on the matrix $A$, which is assumed to have continuous coefficients and a singular set $\mathcal{S}$ of measure zero. On the other hand, we do not assume the a priori validity of local Sobolev inequalities with a strict gain in summability, which may fail in cases where the Poincaré inequality (1.2) holds and our results (and those of [20]) apply. A simple example of this failure is presented in Example 6.6 and hence we cannot always transport the axiomatic program to our setting. As a final comparison, we mention that the Sobolev spaces with matrix valued weight $A$ used in the axiomatic program are defined as completions of locally Lipschitz functions, while we have used $C^{1}$ functions here. Under our assumptions, the two definitions yield the same function spaces as described in Remark 4.9.

The Poincaré inequalities presented here (and in [20]) involve anisotropic matrix valued weights $A$ and do not presume any particular underlying geometric structure, such as a CarnotCarathéodory metric associated to a suitable family $\left\{X_{j}\right\}_{j=1}^{m}$ of locally Lipschitz vector fields on $\Omega$. This underlying sub-Riemannian structure is natural for studying PDEs whose principal part is a sum of squares of the vector fields and has a rich history beginning with the work of Hörmander [17] for smooth vector fields. Hörmander's work, coupled with the seminal papers of Fefferman-Phong [8] in a general nonnegative definite but smooth setting and the first extension to a non-smooth setting in Franchi-Lanconelli [13], also provided the motivation for the Sawyer-Wheeden program descibed above. An underlying sub-Riemmannian structure plays a fundamental role in the analysis on the Heisenberg group and other homogeneous 
Carnot groups (see the monograph of Bonfiglioli, Lanconelli and Uguzzoni [2] for a detailed account). In such a geometric context, there are a wealth of available Poincaré inequalities (see the paper of Hajlasz and Koskela [16] for an extensive survey). While we do not assume such an underlying structure, our class of problems may allow for one provided that the matrix $A$ represents the principal part of the sum of the squares of vector fields $\left\{X_{j}\right\}_{j=1}^{m}$ satisfying the needed sub-Riemannian structural conditions. For example, if $A \in C^{2}\left(\Omega ; \operatorname{Sym}_{n}^{+}(\mathbb{R})\right)$ then the entries of $\sqrt{A}$ will be locally Lipschitz functions (see Theorem 6.1.2 in volume I of [14]) and one could attempt to choose $\left\{X_{j}\right\}_{j=1}^{n}$, where $X_{j}$ is the $j$-th column of $\sqrt{A}$, provided that the resulting degeneracy set $\mathcal{S}$ satisfies the requirements in condition (2.3). We do not require $A$ to be regular enough to support such locally Lipschitz vector fields. On the other hand, for the analysis on homogeneous Carnot groups, the degeneracy set $\mathcal{S}$ does not have to be thin, as we require.

Similar geometric considerations hold for the applications to degenerate PDEs of course. In particular, in the situation described above one sees that $A$ satisfies

$$
\sum_{j=1}^{n}\left\langle X_{j}, \xi\right\rangle^{2}=\langle A(x) \xi, \xi\rangle, \quad \forall x \in \Omega, \xi \in \mathbb{R}^{n},
$$

which means that the principal part $\operatorname{div}\{A(x) \nabla u\}$ is uniformly $X$-elliptic in the language of Lanconelli and Kogoj [18] which rendered explicit the property introduced in Franchi and Lanconelli [12]. In that spirit, we could say that our point of view is merely that $\operatorname{div}\{A(x) \nabla u\}$ is uniformly $\sqrt{A}$-elliptic for any measurable non negative matrix-valued function $A$. Similarly, one could interpret the condition (1.6) in this light by writing

$$
\mu_{1}|\sqrt{A(x)} \xi|^{2} \leq\langle M(x, t) \xi, \xi\rangle \leq \mu_{2}|\sqrt{A(x)} \xi|^{2},
$$

to say that the equation (1.4) is $\sqrt{A}$-parabolic.

With respect to other possible approaches to the degenerate PDEs, we wish to make a few additional remarks. First, we do not assume conditions on the smallest, largest eigenvalues $\lambda(x), \Lambda(x)$ of $A(x)$ of the form

$$
\lambda^{-1} \in L^{p}(\Omega) \text { with } p \geq n / 2
$$

as in Murthy and Stampacchia [24] or

$$
\Lambda(x) \leq C \lambda(x) \text { for some constant } C>0
$$

as in Fabes, Kenig and Serapioni [7], although we will effectively require $\Lambda(x) \leq \Lambda<+\infty$ (see assumption (2.1)-(i)). Similar considerations hold for the degenerate parabolic applications in contrast with the related work of Chiarenza and Serapioni [5]. For the evolution equations, we have taken the variational approach in part in order to easily treat equations with timedependent coefficients, which complicates matters for approaches based on semigroups in this non-autonomous setting. Moreover, we do not fully exploit the unity present on the FicheraOleinik theory of equations with non-negative characteristic form. More precisely, we prefer to treat the degenerate elliptic and degenerate parabolic problems in a distinct but related variational way, making use of the their special features. In principle, one could treat them together (as summarized in Chapter 1 of [25]). However one is able to obtain better parabolic results directly with no additional effort. For example, there are no sign conditions on the coefficient $c(x, t)$ in our parabolic results.

We conclude this introduction with a brief outline of the contents. In section 2 , we present the precise assumptions made on the degenerate diffusion matrix $A$ and reduce the proof of the Poincaré inequality (1.2) to the existence of the aforementioned subsolution pair $w, \widetilde{w}$ (see Proposition 2.1). This proposition uses essentially only the regularity hypotheses (2.1). In section 3 , we complete the proof of (1.2) by constructing the pair $w, \widetilde{w}$ by exploiting the 
structural hypotheses (2.3) on $A$ and $\mathcal{S}$ (especially the normal rank condition). Various mild generalizations and remarks will also be given. Section 4 studies the associated Sobolev spaces with matrix valued weights $A$ under mild assumptions on $A$ and $\mathcal{S}$, but assuming the validity of the Poincaré inequality (1.2) (see hypothesis (4.1)). In section 5 we will apply the present results to degenerate PDEs, where the key point is the validity of (1.2) for the degenerate diffusion matrix $A$. Finally, in section 6 we present the examples mentioned above which illustrate the structural hypotheses made on the weight $A$ and its singular set $\mathcal{S}$ in order to ensure the validity of the Poincaré inequality. In particular, the Poincaré inequality may fail if the orthogonal rank condition of $A$ on $\mathcal{S}$ does not hold, while the Poincaré inequality can be valid without requiring a direction of uniform ellipticity for the diffusion matrix $A$ (as required in [20]) and without assuming that $\mathcal{S}$ has codimension at least two (in accordance with Remark 3.5).

\section{Degenerate diffusion matrices and subsolution pairs}

As described in the introduction, we would like to find sufficient conditions on a degenerate diffusion matrix valued function $A$ on a bounded domain $\Omega \subset \mathbb{R}^{n}$ for which the Poincaré inequality (1.2) holds. In this section, we will give such sufficient conditions and explain how the validity of (1.2) reduces to the existence of a suitable pair of subsolutions to the associated degenerate elliptic operator $\mathcal{L} u:=\operatorname{div}\{A \nabla u\}$. We will first require that $A: \Omega \rightarrow \operatorname{Sym}_{n}^{+}(\mathbb{R})$ is sufficiently regular; more precisely, denoting by $A(x)=\left[a_{i j}(x)\right]_{i, j=1, \ldots, n}$ we will assume that:

$$
\left\{\begin{array}{l}
\text { (i) } a_{i j}=a_{j i} \in C^{1}(\Omega) \cap C^{0}(\bar{\Omega}) \text { for } i, j=1, \ldots, n ; \\
\text { (ii) } \frac{\partial a_{i j}}{\partial x_{j}} \in L^{\infty}(\Omega) \text { for } i, j=1, \ldots, n ; \\
\text { (iii) }\langle\xi, A(x) \xi\rangle=\sum_{i, j=1}^{n} a_{i j}(x) \xi_{i} \xi_{j} \geq 0 \text { for every } x \in \bar{\Omega} \text { and } \xi=\left(\xi_{1}, . ., \xi_{n}\right) \in \mathbb{R}^{n} .
\end{array}\right.
$$

Condition (iii) in (2.1) says that $\mathcal{L}$ is of nonnegative characteristic form and we denote by

$$
\mathcal{S}=\{x \in \Omega \mid \operatorname{det} A(x)=0\}
$$

the degeneracy set of any second order operator having principal part $\mathcal{L}$. Of course, for every $x \in \bar{\Omega} \backslash \mathcal{S}$ one has

$$
\sum_{i, j=1}^{n} a_{i j}(x) \xi_{i} \xi_{j}>0 \text { for any } \xi=\left(\xi_{1}, . ., \xi_{n}\right) \in \mathbb{R}^{n} \backslash\{0\} .
$$

Our assumptions on the domain $\Omega$ and the degeneracy set $\mathcal{S}$ are the following:

(i) $\partial \Omega$ is of class $C^{1,1}$;

(ii) $\mathcal{S}=\bigcup_{\alpha=1}^{m} \mathcal{S}_{\alpha} \subset \Omega$ is the disjoint union of a finite number $m$ of compact connected submanifolds of $\mathbb{R}^{n}$, without boundary, of class $C^{2}$ and having dimension $k_{1}, \ldots, k_{m} \in\{0,1, \ldots, n-2\}$;

(iii) for every point $x_{0} \in \mathcal{S}$ there exists $\zeta=\zeta\left(x_{0}\right) \in \mathbb{R}^{n} \backslash\{0\}$ such that $\zeta \perp \mathcal{S}$ at $x_{0}$ and $\left\langle\zeta, A\left(x_{0}\right) \zeta\right\rangle>0$.

Since $A \geq 0$ as a quadratic form, condition (iii) in (2.3) just says that $A$ restricted to the normal bundle of $\mathcal{S}$ has rank at least one at each point $x_{0} \in \mathcal{S}$. This orthogonal rank may vary from point to point. We will say that $\operatorname{dim} \mathcal{S}_{\alpha}=0$ if $\mathcal{S}_{\alpha}$ is a single point $x_{0}$, and in that case condition (iii) just means that $A\left(x_{0}\right) \neq 0$. Note that, under condition (2.3), the degeneracy set $\mathcal{S}$ has exactly $m$ connected components. 
For $\varepsilon>0$ and $\alpha=1, \ldots, m$ we define the following open neighborhoods of the singular set and its components

$$
\mathcal{S}^{\varepsilon}:=\{x \in \Omega \mid d(x, \mathcal{S})<\varepsilon\} \quad \text { and } \quad \mathcal{S}_{\alpha}^{\varepsilon}:=\left\{x \in \Omega \mid d\left(x, \mathcal{S}_{\alpha}\right)<\varepsilon\right\} .
$$

We always assume that $\varepsilon>0$ is small enough to ensure that

$$
\overline{\mathcal{S}^{\varepsilon}} \subset \Omega \quad \text { and } \quad \overline{\mathcal{S}_{\alpha}^{\varepsilon}} \cap \overline{\mathcal{S}_{\beta}^{\varepsilon}}=\emptyset \text { for } \alpha \neq \beta .
$$

By the structural condition ( $i i)$ of $(2.3)$, all of the connected components $\mathcal{S}_{1}, \ldots, \mathcal{S}_{m}$ of $\mathcal{S}$ have codimension at least two. This hypothesis combined with (2.4) implies that

$$
\Omega \backslash \overline{\mathcal{S}^{\varepsilon}} \text { is connected }
$$

since $\Omega$ is connected. Indeed, by (2.4), one can remove one connected component of $\overline{\mathcal{S}^{\varepsilon}}$ at a time. Exploiting a bundle of planes orthogonal to $\mathcal{S}_{\alpha}$, one easily shows that $\Omega \backslash \overline{\mathcal{S}_{\alpha}^{\varepsilon}}$ is path connected. Moreover, by choosing $\varepsilon>0$ even smaller if necessary, we can assume that each of the open sets $\mathcal{S}_{\alpha}^{\varepsilon}$ is of class $C^{2}$, see for instance [11, Theorem 1].

Making use of the elementary inequality

$$
\left|\left\langle M v_{1}, v_{2}\right\rangle\right| \leq\left|\sqrt{M} v_{1}\right|\left|\sqrt{M} v_{2}\right|, \quad M \in \operatorname{Sym}_{n}^{+}(\mathbb{R}), v_{1}, v_{2} \in \mathbb{R}^{n}
$$

and performing simple $L^{p}$ estimates for degenerate elliptic equations along the lines of Fichera (see section 3 of [10] as well as Lemma 1.2.1 of [25]), one has the following sufficient conditions for the validity of the Poincaré inequality (1.2) in terms of the existence of a suitable pair of local strict subsolutions $w, \widetilde{w}$.

Proposition 2.1 Let $A$ satisfy the conditions (2.1) and let $p \in[1, \infty)$. Assume that $\mathcal{S} \subset \Omega$, that $\Theta$ is a $C^{1,1}$ bounded open set such that $\mathcal{S} \subset \Theta \subset \bar{\Theta} \subset \Omega$ and that there exist $w \in C^{2}(\bar{\Theta})$, $\widetilde{w} \in C^{1}(\overline{\Omega \backslash \Theta}) \cap W^{2, p}(\Omega \backslash \Theta)$ for some $p \geq 1, \theta>0, K>0$ such that

$$
\begin{aligned}
& \operatorname{div}(A(x) \nabla w) \geq \theta \quad \text { in } \Theta, \\
& |\sqrt{A(x)} \nabla w| \leq K \quad \text { in } \Theta, \\
& \operatorname{div}(A(x) \nabla \widetilde{w}) \geq \theta \quad \text { a.e. in } \Omega \backslash \bar{\Theta}, \\
& |\sqrt{A(x)} \nabla \widetilde{w}| \leq K \quad \text { in } \Omega \backslash \bar{\Theta} .
\end{aligned}
$$

Furthermore, suppose that

$$
\langle A(x)(\nabla w-\nabla \widetilde{w}), \hat{\nu}\rangle \leq 0 \quad \text { on } \partial \Theta,
$$

where $\hat{\nu}$ denotes the outward unit normal to $\partial \Theta$. Then, for every $u \in C_{0}^{1}(\Omega)$, we have

$$
\|u\|_{L^{p}(\Omega)} \leq C\left(\int_{\Omega}\langle A(x) \nabla u, \nabla u\rangle^{\frac{p}{2}} d x\right)^{\frac{1}{p}},
$$

for some constant $C>0$ depending only on $p, \theta, K$.

Proof. Let $\delta>0$ and $u \in C_{0}^{1}(\Omega)$. By the Divergence Theorem, since $\operatorname{supp} u \subset \Omega, A(x) \nabla \widetilde{w} \in$ $W^{1, p}(\Omega \backslash \Theta)$ and by the regularity of $u$ and of $\partial \Theta$, we obtain

$$
\begin{aligned}
\int_{\Theta} \operatorname{div} & (A(x) \nabla w)\left(u^{2}+\delta\right)^{\frac{p}{2}} d x+\int_{\Omega \backslash \Theta} \operatorname{div}(A(x) \nabla \widetilde{w})\left(u^{2}+\delta\right)^{\frac{p}{2}} d x= \\
& -p \int_{\Theta}\langle A(x) \nabla w, \nabla u\rangle u\left(u^{2}+\delta\right)^{\frac{p}{2}-1} d x+\int_{\partial \Theta}\left\langle A(x) \nabla w, \nu_{\varepsilon}\right\rangle\left(u^{2}+\delta\right)^{\frac{p}{2}} d \sigma \\
& -p \int_{\Omega \backslash \Theta}\langle A(x) \nabla \widetilde{w}, \nabla u\rangle u\left(u^{2}+\delta\right)^{\frac{p}{2}-1} d x-\int_{\partial \Theta}\left\langle A(x) \nabla \widetilde{w}, \nu_{\varepsilon}\right\rangle\left(u^{2}+\delta\right)^{\frac{p}{2}} d \sigma \\
& +\int_{\partial \Omega}\langle A(x) \nabla \widetilde{w}, \nu\rangle\left(u^{2}+\delta\right)^{\frac{p}{2}} d x,
\end{aligned}
$$


where $\nu$ denotes the outward unitary normal vector to $\partial \Omega$. Letting $\delta \rightarrow 0^{+}$in (2.13), by the Dominated Convergence Theorem and recalling that $\operatorname{supp} u \subset \Omega$, and thus $u=0$ on $\partial \Omega$,

$$
\begin{aligned}
& \int_{\Theta} \operatorname{div}(A(x) \nabla w)|u|^{p} d x+\int_{\Omega \backslash \Theta} \operatorname{div}(A(x) \nabla \widetilde{w})|u|^{p} d x= \\
& \quad-p \int_{\Theta}\langle A(x) \nabla w, \nabla u\rangle u|u|^{p-2} d x-p \int_{\Omega \backslash \Theta}\langle A(x) \nabla \widetilde{w}, \nabla u\rangle u|u|^{p-2} d x \\
& \quad+\int_{\partial \Theta}\left\langle A(x)\left(\nabla w-\nabla \widetilde{w}, \nu_{\varepsilon}\right\rangle|u|^{p} d \sigma .\right.
\end{aligned}
$$

Here, in case $p=1$, we have $u|u|^{-1}=\operatorname{sgn} u$ with the convention that the function is 0 at each point of the domain where $u=0$. From (2.14), using conditions (2.7), (2.9), (2.11) and inequality (2.6), we get

$$
\begin{aligned}
\theta \int_{\Omega}|u|^{p} d x \leq & p \int_{\Theta}|\sqrt{A(x)} \nabla w||\sqrt{A(x)} \nabla u||u|^{p-1} d x \\
& +p \int_{\Omega \backslash \Theta}|\sqrt{A(x)} \nabla \widetilde{w}||\sqrt{A(x)} \nabla u||u|^{p-1} d x .
\end{aligned}
$$

By (2.15), (2.8) and (2.10) we obtain

$$
\theta \int_{\Omega}|u|^{p} d x \leq p K \int_{\Omega}|\sqrt{A}(x) \nabla u||u|^{p-1} d x .
$$

If $p=1$, the conclusion now easily follows by dividing through by $\theta>0$. In case $p>1$, in view of Hölder inequality, (2.16) yields

$$
\int_{\Omega}|u|^{p} d x \leq \frac{p K}{\theta}\left(\int_{\Omega}\langle A(x) \nabla u, \nabla u\rangle^{\frac{p}{2}} d x\right)^{\frac{1}{p}}\left(\int_{\Omega}|u|^{p} d x\right)^{\frac{p-1}{p}} .
$$

This easily implies the conclusion with $C=\frac{p K}{\theta}$.

We conclude this section by noting that that the parameters $\theta$ and $K$ will depend, in practice, on the domain $\Omega$ and the coefficients of $A$ and hence $C=C(p, \Omega, A)$. This will be illustrated in the following section when we construct the pair $w, \widetilde{w}$ and the neighborhood $\Theta$ of $\mathcal{S}$.

\section{The Poincaré inequality with matrix valued weights}

In this section, we will construct the subsolution pair $w, \widetilde{w}$ required by Proposition 2.1 and hence complete the proof of the following Poincaré inequality with matrix valued weight $A$.

Theorem 3.1 Assume that $A$ and $\Omega$ satisfy the assumptions (2.1) and (2.3) and let $p \in$ $[1, \infty)$. Then there exists a constant $C_{\mathcal{P}}=C_{\mathcal{P}}(p, \Omega, A)>0$ such that for every $u \in C_{0}^{1}(\Omega)$ one has

$$
\|u\|_{L^{p}(\Omega)} \leq C_{\mathcal{P}}\left(\int_{\Omega}\langle A(x) \nabla u, \nabla u\rangle^{\frac{p}{2}} d x\right)^{\frac{1}{p}} .
$$

Before giving the proof, we wish to make a few remarks about extending the applicability of the theorem. A first obvious consequence of Theorem 3.1 allows for degeneracy on the boundary on the boundary of a domain $\Omega^{\prime}$, provided that it has compact closure within a domain on which the theorem applies. In particular, no regularity hypotheses on $\partial \Omega^{\prime}$ need to be made as one merely extends $u$ to be zero on the complement $\bar{\Omega} \backslash \Omega^{\prime}$ and uses the regularity on $\partial \Omega$. 
Remark 3.2 If the conditions (2.1) and (2.3) are satisfied by $A$ on $\Omega$ and $p \in[1, \infty)$, then for each subdomain $\Omega^{\prime} \Subset \Omega$ there exists a constant $C=C\left(p, A, \Omega^{\prime}\right)>0$ such that

$$
\|u\|_{L^{p}\left(\Omega^{\prime}\right)} \leq C\left(\int_{\Omega^{\prime}}\langle A(x) \nabla u, \nabla u\rangle^{\frac{p}{2}} d x\right)^{\frac{1}{p}}
$$

holds for each $u \in C_{0}^{1}\left(\Omega^{\prime}\right)$.

Next we note that one can relax the regularity assumptions on the coefficients of the matrix valued weight if it dominates a weight for which the theorem applies.

Remark 3.3 Assume that conditions (2.1) and (2.3) are satisfied by $A$ on $\Omega$ and that $p \in$ $[1, \infty)$. If $B: \Omega \rightarrow \operatorname{Sym}_{n}^{+}(\mathbb{R})$ is measurable and admits $C_{0}>0$ such that

$$
\langle\xi, A(x) \xi\rangle \leq C_{0}\langle\xi, B(x) \xi\rangle
$$

for a.e. $x \in \Omega$ and every $\xi \in \mathbb{R}^{n}$, then there exists a constant $C>0$ such that for every $u \in C_{0}^{1}(\Omega)$ one has

$$
\|u\|_{L^{p}(\Omega)} \leq C\left(\int_{\Omega}\langle B(x) \nabla u, \nabla u\rangle^{\frac{p}{2}} d x\right)^{\frac{1}{p}} .
$$

Finally, we note two possible ways in which one can relax the assumptions on the degeneracy set $\mathcal{S}$. The first concerns the regularity of $\mathcal{S}$, while the second concerns the codimension of $\mathcal{S}$.

Remark 3.4 As the proof of Theorem 3.1 shows, the Poincaré inequality (3.1) will hold on $\Omega$ provided that one has

i) the regularity condition (2.1),

ii) $\partial \Omega$ is of class $C^{1,1}$,

iii) $\mathcal{S} \subset \Omega$ and $\Omega \backslash \overline{\mathcal{S}^{\varepsilon}}$ has only one connected component for $\varepsilon>0$ small enough,

iv) for every $x_{0} \in \mathcal{S}$ there exists a closed ball $\bar{B}_{R}\left(x_{0}\right) \subset \Omega$ and a function $\Phi \in C^{2}\left(\bar{B}_{R}\left(x_{0}\right)\right)$ such that

$$
\mathcal{S} \cap \bar{B}_{R}\left(x_{0}\right) \subset\left\{x \in \bar{B}_{R}\left(x_{0}\right) \mid \Phi(x)=0\right\}
$$

and $\left\langle\nabla \Phi\left(x_{0}\right), A\left(x_{0}\right) \nabla \Phi\left(x_{0}\right)\right\rangle>0$.

See also Proposition 3.6. These conditions may hold in some cases, even when the degeneracy set $\mathcal{S}$ fails to be of class $C^{2}$, as required in condition (2.3).

Remark 3.5 The case when the degeneracy set $\mathcal{S}$ has at least one connected component of dimension $n-1$ is more delicate, but we can recover the result under an additional technical hypothesis. Indeed, as the proof of Theorem 3.1 will show, if one assumes (2.1) and relaxes (2.3) to allow $k_{1}, \ldots, k_{m}$ to vary in $\{0,1, \ldots, n-1\}$, then (3.1) still holds, provided that one assumes

$$
\partial \Omega_{i} \cap \partial \Omega \neq \emptyset \quad \text { for all } i=1, \ldots, n_{0}
$$

where $\Omega_{i}$ are the connected components of $\Omega \backslash \mathcal{S}$; that is,

$$
\Omega \backslash \mathcal{S}=\bigcup_{i=1}^{n_{0}} \Omega_{i},
$$

for some $n_{0} \in \mathbb{I N}$. More precisely, this condition can be used in Step III of the proof in order to obtain existence of a solution to problem (3.26), in every connected component $\Omega_{i}$.

We are now ready to discuss the proof. As explained in section 2, Theorem 3.1 follows from Proposition 2.1, provided that we can exhibit a suitable subsolution pair $w, \widetilde{w}$. Their construction will be achieved in three steps: 
I. For any $x_{0} \in \mathcal{S}$, construct a function $\varphi \in C^{2}\left(\bar{B}_{R}\left(x_{0}\right)\right)$, with $\bar{B}_{R}\left(x_{0}\right) \subset \Omega$, such that

$$
\operatorname{div}(A(x) \nabla \varphi) \geq \theta \quad \text { in } B_{R}\left(x_{0}\right)
$$

and

$$
|\sqrt{A(x)} \nabla \varphi| \leq K \quad \text { in } B_{R}\left(x_{0}\right),
$$

for some $R, K, \theta>0$, depending on $x_{0}$.

II. Define $\Theta=\mathcal{S}^{\varepsilon}$ and, by using the compactness of $\mathcal{S}$ and Step I above, construct a function $w$ satisfying (2.7) and (2.8) on $\Theta=\mathcal{S}^{\varepsilon}$ for $\varepsilon>0$ small enough;

III. Exploit the uniform ellipticity away from the degeneracy set $\mathcal{S}$ to construct the function $\widetilde{w}$ such that (2.9), (2.10) and (2.11) are satisfied, with $\Theta=\mathcal{S}^{\varepsilon}$ as in Step II above.

\subsection{Step I of the proof of Theorem 3.1}

We begin with a local construction near the singular set $\mathcal{S}$.

Proposition 3.6 Let the assumptions of Theorem 3.1 be satisfied and let $x_{0} \in \mathcal{S}$. Then there exist $R, K, \theta>0$ and $\Phi \in C^{2}\left(\bar{B}_{R}\left(x_{0}\right)\right)$ with $\bar{B}_{R}\left(x_{0}\right) \subset \Omega$ such that

i) $\varphi=\Phi^{2}$ satisfies (3.3) and (3.4); that is,

$$
\operatorname{div}(A(x) \nabla \varphi) \geq \theta \quad \text { and } \quad|\sqrt{A(x)} \nabla \varphi| \leq K \quad \text { in } B_{R}\left(x_{0}\right)
$$

ii) one has

$$
R<\frac{1}{3} \min _{\alpha \neq \beta} d\left(\mathcal{S}_{\alpha}, \mathcal{S}_{\beta}\right)
$$

iii) one has

$$
\mathcal{S} \cap \bar{B}_{R}\left(x_{0}\right) \subset\left\{x \in \bar{B}_{R}\left(x_{0}\right) \mid \Phi(x)=0\right\} .
$$

Remark 3.7 Since $\mathcal{S}_{1}, \ldots, \mathcal{S}_{m}$ are compact, $\min _{\alpha \neq \beta} d\left(\mathcal{S}_{\alpha}, \mathcal{S}_{\beta}\right)$ is finite and positive. In particular, if $x_{0}, x_{1} \in \mathcal{S}$ belong to different connected components of $\mathcal{S}$, the corresponding balls provided by Proposition 3.6 have closures which do not intersect.

Proof. Since $x_{0} \in \mathcal{S}$, there exists a unique $\alpha \in\{1, \ldots, m\}$ such that $x_{0} \in \mathcal{S}_{\alpha}$. Let $\zeta \in \mathbb{R}^{n} \backslash\{0\}$ be such that $\zeta \perp \mathcal{S}$ at $x_{0}$ and $\left\langle\zeta, A\left(x_{0}\right) \zeta\right\rangle>0$, as given by the normal rank condition (iii) of (2.3). We construct $\Phi$ in terms of this direction $\zeta$ along which $A$ is nondegenerate.

Claim: There exist $r>0$ and $\Phi \in C^{2}\left(\bar{B}_{r}\left(x_{0}\right)\right)$ such that $\bar{B}_{r}\left(x_{0}\right) \subset \Omega$,

$$
\mathcal{S} \cap \bar{B}_{r}\left(x_{0}\right) \subset\left\{x \in \bar{B}_{r}\left(x_{0}\right) \mid \Phi(x)=0\right\},
$$

$\bar{B}_{r}\left(x_{0}\right) \cap \mathcal{S}_{\beta}=\emptyset$ if $\beta \neq \alpha$ and $\nabla \Phi\left(x_{0}\right)=\zeta$.

Indeed, in the case when $\mathcal{S}_{\alpha}=\left\{x_{0}\right\}$, first choose $r>0$ small enough so that $\bar{B}_{r}\left(x_{0}\right) \subset \Omega$ and $\bar{B}_{r}\left(x_{0}\right) \cap \mathcal{S}_{\beta}=\emptyset$ for every $\beta \neq \alpha$. This is possible by the compactness of $\mathcal{S}_{1}, \ldots, \mathcal{S}_{m}$ and since $x_{0} \notin \mathcal{S}_{\beta}$ for $\beta \neq \alpha$. Then we can choose $\Phi(x):=\left\langle x-x_{0}, \zeta\right\rangle$ on $\bar{B}_{r}\left(x_{0}\right)$ and the claim follows in this case.

In the remaining cases, since $\mathcal{S}_{\alpha}$ is a $C^{2}$ manifold of dimension $k_{\alpha}>0$ in $\mathbb{R}^{n}$, we can find an open neighborhood $W \subset \mathbb{R}^{n}$ of $x_{0}$ and a function $F \in C^{2}\left(W, \mathbb{R}^{n-k_{\alpha}}\right)$ such that

$$
W \cap \mathcal{S}_{\alpha}=\{x \in W \mid F(x)=0\}, \quad \operatorname{rk}[D F(x)]=n-k_{\alpha} \text { for every } x \in W .
$$

Since $\mathcal{S}_{1}, \ldots \mathcal{S}_{m} \subset \Omega$ are compact and disjoint, by choosing a smaller neighborhood $W$ if need be, we can assume that $W \subset \Omega$ and that $W \cap \mathcal{S}_{\beta}=\emptyset$ for $\beta \neq \alpha$.

Let $F_{1}, \ldots, F_{n-k_{\alpha}}$ denote the scalar components of $F$. By applying condition (3.9), we have that $F_{j}=0$ and $\nabla F_{j} \neq 0$ on $W \cap \mathcal{S}_{\alpha}$ for every $j=1, \ldots, n-k_{\alpha}$. Thus $\nabla F_{j}(x)$ is perpendicular 
to $\mathcal{S}_{\alpha}$ at $x$, for every $x \in W \cap \mathcal{S}_{\alpha}$ and every $j=1, \ldots, n-k_{\alpha}$. By condition (3.9) the vectors $\nabla F_{1}(x), \ldots, \nabla F_{n-k_{\alpha}}(x)$ are independent for every $x \in W \cap \mathcal{S}_{\alpha}$. Since $\mathcal{S}_{\alpha} \subset \mathbb{R}^{n}$ has dimension $k_{\alpha}$, for every $x \in \mathcal{S}_{\alpha} \cap W$ the system $\left\{\nabla F_{j}(x)\right\}_{j=1}^{n-k_{\alpha}}$ form a basis of the space of vectors in $\mathbb{R}^{n}$ that are perpendicular to $\mathcal{S}_{\alpha}$ at $x$. Since $\zeta \perp \mathcal{S}$ at $x_{0} \in \mathcal{S}_{\alpha}$, we have

$$
\zeta=\sum_{j=1}^{n-k_{\alpha}} c_{j} \nabla F_{j}\left(x_{0}\right)
$$

for some constants $c_{1}, \ldots, c_{n-k_{\alpha}} \in \mathbb{R}$. Now let $r>0$ be small enough so that $\bar{B}_{r}\left(x_{0}\right) \subset W$; then the function

$$
\Phi(x):=\sum_{j=1}^{n-k_{\alpha}} c_{j} F_{j}(x)
$$

satisfies all the requirements in the claim.

Having selected $\Phi$, we need only verify that $R, K, \theta$ can be found so that (3.5) - (3.7) hold. By condition (iii) of (2.3), we have

$$
\left\langle\nabla \Phi\left(x_{0}\right), A\left(x_{0}\right) \nabla \Phi\left(x_{0}\right)\right\rangle=\left\langle\zeta, A\left(x_{0}\right) \zeta\right\rangle:=2 \theta>0 ;
$$

which selects $\theta$. Using the continuity of $A$ and $\nabla \Phi$ and choosing $r>0$ smaller if need be, we can assume that

$$
\langle\nabla \Phi(x), A(x) \nabla \Phi(x)\rangle \geq \theta>0, \quad \text { in } \bar{B}_{r}\left(x_{0}\right) .
$$

For $x \in B_{r}\left(x_{0}\right)$, we compute the expression needed for the first property in (3.5):

$$
\begin{aligned}
\operatorname{div}\left(A(x) \nabla\left(\Phi^{2}(x)\right)\right)= & 2 \Phi(x) \sum_{i, j=1}^{n}\left(\frac{\partial a_{i j}}{\partial x_{j}}(x) \frac{\partial \Phi}{\partial x_{i}}(x)+a_{i j}(x) \frac{\partial^{2} \Phi}{\partial x_{i} \partial x_{j}}(x)\right) \\
& +2\langle\nabla \Phi(x), A(x) \nabla \Phi(x)\rangle .
\end{aligned}
$$

Since $A \in C^{1}\left(\bar{B}_{r}\left(x_{0}\right)\right)$ and $\Phi \in C^{1}\left(\bar{B}_{r}\left(x_{0}\right)\right)$, there exists a constant $M>0$ such that

$$
\left|2 \sum_{i, j=1}^{n} \frac{\partial a_{i j}}{\partial x_{j}}(x) \frac{\partial \Phi}{\partial x_{i}}(x)+a_{i j}(x) \frac{\partial^{2} \Phi}{\partial x_{i} \partial x_{j}}(x)\right| \leq M \quad \text { in } \bar{B}_{r}\left(x_{0}\right) .
$$

Since $\Phi\left(x_{0}\right)=0$ and is continuous, we can choose $R \in(0, r)$ small enough so that (3.6) holds and

$$
|\Phi(x)| \leq \frac{\theta}{M} \quad \text { in } \bar{B}_{R}\left(x_{0}\right) .
$$

Setting $\varphi=\Phi^{2}$, we have $\varphi \in C^{2}\left(\bar{B}_{R}\left(x_{0}\right)\right)$ and hence one has the existence of a constant $K>0$ for which the second property in (3.5) holds. Moreover, by (3.10)-(3.13), we easily obtain

$$
\operatorname{div}(A(x) \nabla \varphi(x)) \geq \theta>0
$$

on $\bar{B}_{R}\left(x_{0}\right)$, which gives the first property in (3.5). Finally, condition (3.7) is an immediate consequence of $(3.8)$ since $0<R<r$.

\subsection{Step II of the proof of Theorem 3.1}

Using Proposition 3.6, we now construct a function $w \in C^{2}\left(\overline{\mathcal{S}^{\varepsilon}}\right)$ satisfying (2.7) and (2.8) on $\Theta=\mathcal{S}^{\varepsilon}$, for $\varepsilon>0$ small enough. The function $w$ will be constructed separately on a neighborhood $\mathcal{S}_{\alpha}^{\varepsilon}$ of each $\mathcal{S}_{\alpha}$ for every fixed $\alpha=1, \ldots, m$, with $\varepsilon>0$ small enough so that (2.4) holds. 
Fix $\alpha=1, \ldots, m$. If $\mathcal{S}_{\alpha}=\left\{x_{0}\right\}$ has dimension 0 , then the function $w=\Phi^{2}$ constructed using Proposition 3.6 satisfies (2.7) and (2.8) in $\mathcal{S}_{\alpha}^{\varepsilon}=B_{\varepsilon}\left(x_{0}\right)$, for $\varepsilon>0$ small enough. Hence we can restrict our attention to those $\alpha$ for which $\operatorname{dim}\left(\mathcal{S}_{\alpha}\right)>0$.

Using the compactness of $\mathcal{S}_{\alpha}$ and Proposition 3.6, we can cover $\mathcal{S}_{\alpha}$ by a finite number $N=N(\alpha)$ of balls

$$
\mathcal{S}_{\alpha} \subset \bigcup_{k=1}^{N} B_{R_{k}}\left(x_{k}\right):=\Omega_{\alpha}^{*}
$$

with centers $x_{k} \in \mathcal{S}_{\alpha}$ and radii satisfying

$$
R_{k}<\frac{1}{3} d\left(\mathcal{S}_{\alpha}, \mathcal{S}_{\beta}\right) \quad \text { for each } \beta \neq \alpha
$$

for which there are functions $\Phi_{k} \in C^{2}\left(\bar{B}_{R_{k}}\left(x_{k}\right)\right)$ satisfying (3.5) and (3.7) with $R_{k}, \Phi_{k}, x_{k}$ in place of $R, \Phi, x_{0}$.

In order to complete the construction of the subsolution $w$ in a neighborhood of $\mathcal{S}$, we note that distinct connected components $\mathcal{S}_{\alpha}$ have associated coverings $\Omega_{\alpha}^{*}$ with disjoint closures. Hence it will suffice to introduce a suitable partition of unity subordinate to the open covering $\left\{B_{R_{k}}\left(x_{k}\right)\right\}_{k=1}^{N}$ of each connected component $\mathcal{S}_{\alpha}$ of $\mathcal{S}$ in order to patch together the family of functions $\left\{\Phi_{k}^{2}\right\}_{k=1}^{N}$. To this end, we note that for each fixed $\alpha$ the neighborhood $\Omega_{\alpha}^{*}$ is open and satisfies $\bar{\Omega}_{\alpha}^{*} \subset \Omega$. In particular, $d\left(\mathcal{S}_{\alpha}, \partial \Omega_{\alpha}^{*}\right)>0$; thus there exists $\varepsilon_{*}>0$ such that

$$
\overline{\mathcal{S}_{\alpha}^{\varepsilon_{*}}}=\left\{x \in \Omega \mid d\left(x, \mathcal{S}_{\alpha}\right) \leq \varepsilon_{*}\right\} \subset \Omega_{\alpha}^{*} ;
$$

that is, $\left\{B_{R_{k}}\left(x_{k}\right)\right\}_{k=1}^{N}$ is also an open covering of the fattening $\overline{\mathcal{S}_{\alpha}^{\varepsilon_{*}}}$ of the component $\mathcal{S}_{\alpha}$. We may then select a smooth partition of unity $\left\{\psi_{k}\right\}_{k=1}^{N}$ on $\overline{\mathcal{S}_{\alpha}^{\varepsilon_{*}^{*}}}$ which is subordinate to the open covering $\left\{B_{R_{k}}\left(x_{k}\right)\right\}_{k=1}^{N}$; that is,

$$
\begin{aligned}
& \psi_{k} \in C_{0}^{\infty}\left(B_{R_{k}}\left(x_{k}\right)\right), \quad \psi_{k} \geq 0 \quad \text { for } k=1, \ldots, N, \\
& \sum_{k=1}^{N} \psi_{k}(x)=1 \quad \text { for every } x \in \overline{\mathcal{S}_{\alpha}^{\varepsilon_{*}}} .
\end{aligned}
$$

Since supp $\psi_{k}$ is compact and is contained in $B_{R_{k}}\left(x_{k}\right)$, one has

$$
\gamma_{k}:=d\left(\operatorname{supp} \psi_{k}, \partial B_{R_{k}}\left(x_{k}\right)\right)>0 \quad \text { for } k=1, \ldots, N .
$$

Moreover, using the regularity of $\psi_{k}, \Phi_{k}$ and the coefficients $a_{i j}$, there exists a constant $M=$ $M(\alpha)>0$ such that

$$
\begin{aligned}
& \left|\psi_{k}(x)\right| \leq M, \quad\left|\frac{\partial \psi_{k}}{\partial x_{j}}(x)\right| \leq M, \quad\left|\frac{\partial^{2} \psi_{k}}{\partial x_{i} \partial x_{j}}(x)\right| \leq M, \\
& \left|a_{i j}(x)\right| \leq M, \quad\left|\frac{\partial a_{i j}}{\partial x_{j}}(x)\right| \leq M, \quad\left|\frac{\partial \Phi_{k}}{\partial x_{j}}(x)\right| \leq M
\end{aligned}
$$

for every $x \in \bar{\Omega}_{\alpha}^{*}$, every $k=1, \ldots, N$ and every $i, j=1, \ldots, n$. In particular, from the last inequality in (3.16) it follows that

$$
\left|\Phi_{k}(x)-\Phi_{k}(y)\right| \leq \sqrt{n} M|x-y|
$$

for every $k=1, \ldots, N$ and every $x, y \in B_{R_{k}}\left(x_{k}\right)$. By Proposition 3.6 , for every $k=1, \ldots, N$ there exists $\theta_{k}>0$ such that

$$
\operatorname{div}\left(A(x) \nabla\left(\Phi_{k}^{2}(x)\right)\right) \geq \theta_{k} \quad \text { on } B_{R_{k}}\left(x_{k}\right) .
$$


Finally, we pick $\varepsilon$ and $\theta$ to satisfy

$$
0<\varepsilon<\min \left\{\varepsilon_{*}, \gamma_{1}, \ldots, \gamma_{N}\right\}, \quad \theta=\frac{1}{2} \min \left\{\theta_{1}, \ldots, \theta_{N}\right\}
$$

and on $\overline{\mathcal{S}_{\alpha}^{\varepsilon}}$ we define the function

$$
w(x)=\sum_{k=1}^{N} \psi_{k}(x) \Phi_{k}^{2}(x)
$$

This completes the construction of $w$.

It remains only to verify that $w$ satisfies the required properties. By construction, we have $w \in C^{2}\left(\overline{\mathcal{S}_{\alpha}^{\varepsilon}}\right)$ and

$$
\begin{aligned}
\operatorname{div}(A(x) \nabla w(x))= & \sum_{k=1}^{N} \psi_{k}(x) \operatorname{div}\left(A(x) \nabla\left(\Phi_{k}^{2}(x)\right)\right) \\
& +\sum_{k=1}^{N} 4 \Phi_{k}(x)\left\langle\nabla \Phi_{k}(x), A(x) \nabla \psi_{k}(x)\right\rangle+\Phi_{k}^{2}(x) \operatorname{div}\left(A(x) \nabla \psi_{k}(x)\right)
\end{aligned}
$$

for every $x \in \overline{\mathcal{S}_{\alpha}^{\varepsilon}}$. Using conditions (3.14) and (3.18), since $\overline{\mathcal{S}_{\alpha}^{\varepsilon}} \subset \mathcal{S}_{\alpha}^{\varepsilon_{*}} \subset \Omega_{\alpha}^{*}$, we easily see that

$$
\sum_{k=1}^{N} \psi_{k}(x) \operatorname{div}\left(A(x) \nabla\left(\Phi_{k}^{2}(x)\right)\right) \geq \sum_{k=1}^{N} \psi_{k}(x) \theta_{k} \geq 2 \theta>0 \quad \text { on } \mathcal{S}_{\alpha}^{\varepsilon} .
$$

Moreover, for every $x \in \mathcal{S}_{\alpha}^{\varepsilon}$ the conditions in (3.16) yield

$$
\begin{aligned}
& \mid \sum_{k=1}^{N} \Phi_{k}^{2}(x) \operatorname{div}\left(A(x) \nabla \psi_{k}(x)\right) \mid \\
&=\left|\sum_{k=1}^{N} \Phi_{k}^{2}(x)\left(\sum_{i, j=1}^{n} \frac{\partial a_{i j}}{\partial x_{j}}(x) \frac{\partial \psi_{k}}{\partial x_{i}}(x)+a_{i j}(x) \frac{\partial^{2} \psi_{k}}{\partial x_{i} \partial x_{j}}(x)\right)\right| \\
& \leq 2 n^{2} M^{2} \sum_{k=1}^{N} \Phi_{k}^{2}(x) \chi_{\operatorname{supp} \psi_{k}}(x),
\end{aligned}
$$

where $\chi_{A}$ is the characteristic function of the set $A$. If $\chi_{\operatorname{supp} \psi_{k}}(x) \neq 0$, then $x \in \operatorname{supp} \psi_{k} \subset$ $B_{R_{k}}\left(x_{k}\right)$, and hence

$$
d\left(x, \partial B_{R_{k}}\left(x_{k}\right)\right) \geq d\left(\operatorname{supp} \psi_{k}, \partial B_{R_{k}}\left(x_{k}\right)\right)=\gamma_{k}>\varepsilon .
$$

On the other hand, $d\left(x, \mathcal{S}_{\alpha}\right)<\varepsilon$, since $x \in \mathcal{S}_{\alpha}^{\varepsilon}$; in particular, there exists $x_{*} \in \mathcal{S}_{\alpha} \cap B_{\varepsilon}(x)$. Condition (3.22) shows that $B_{\varepsilon}(x) \subset B_{R_{k}}\left(x_{k}\right)$ and hence $x_{*} \in \mathcal{S}_{\alpha} \cap B_{R_{k}}\left(x_{k}\right)$. Using condition (3.7) of Proposition 3.6, we conclude that $\Phi_{k}\left(x_{*}\right)=0$, and hence by (3.17)

$$
\left|\Phi_{k}(x)\right|=\left|\Phi_{k}(x)-\Phi_{k}\left(x_{*}\right)\right| \leq \sqrt{n} M\left|x-x_{*}\right|<\sqrt{n} M \varepsilon .
$$

Combining (3.21) and (3.23) we obtain

$$
\left|\sum_{k=1}^{N} \Phi_{k}^{2}(x) \operatorname{div}\left(A(x) \nabla \psi_{k}(x)\right)\right| \leq 2 n^{3} N M^{4} \varepsilon^{2} \quad \text { for every } x \in \mathcal{S}_{\alpha}^{\varepsilon} .
$$

With a similar argument, using again inequality (3.23), it is easy to see that

$$
\left|4 \sum_{k=1}^{N} \Phi_{k}(x)\left\langle\nabla \Phi_{k}(x), A(x) \nabla \psi_{k}(x)\right\rangle\right| \leq 4 n^{\frac{5}{2}} N M^{4} \varepsilon \quad \text { for every } x \in \mathcal{S}_{\alpha}^{\varepsilon} .
$$


Combining (3.20), (3.24) and (3.25) with (3.19) we see that

$$
\operatorname{div}(A(x) \nabla w(x)) \geq 2 \theta-4 n^{\frac{5}{2}} N M^{4} \varepsilon-2 n^{3} N M^{4} \varepsilon^{2} \geq \theta>0
$$

for every $x \in \mathcal{S}_{\alpha}^{\varepsilon}$, if $\varepsilon>0$ is small enough. Hence $w$ satisfies $(2.7)$ on $\overline{\mathcal{S}_{\alpha}^{\varepsilon}}$; by the compactness of $\overline{\mathcal{S}_{\alpha}^{\varepsilon}}$ and by regularity of the entries of the matrix $A(x)$ and of the function $w$, condition $(2.8)$ is also trivially satisfied for some positive $K>0$ on $\overline{\mathcal{S}_{\alpha}^{\varepsilon}}$.

Repeating the above argument on each connected component $\overline{\mathcal{S}_{1}^{\varepsilon}}, \ldots, \overline{\mathcal{S}_{m}^{\varepsilon}}$ of $\overline{\mathcal{S}^{\varepsilon}}$ we obtain the function $w$ with the desired properties.

\subsection{Step III of the proof of Theorem 3.1}

Exploiting the uniform ellipticity of $\operatorname{div}(A(x) \nabla v)$ away from the degeneracy set $\mathcal{S}$, we now proceed to construct a function $\widetilde{w} \in C^{1}\left(\overline{\Omega \backslash \mathcal{S}^{\varepsilon}}\right) \cap W^{2, p}\left(\Omega \backslash \mathcal{S}^{\varepsilon}\right)$ which satisfies conditions (2.9), (2.10) and (2.11) for some $\theta, K>0$ and for $\varepsilon>0$ small enough.

We start by considering the function $w$ constructed in Step II of the proof. Up to choosing a smaller $\varepsilon>0$, we can assume that $w \in C^{2}\left(\overline{\mathcal{S}^{2 \varepsilon}}\right)$ and that $\overline{\mathcal{S}^{2 \varepsilon}} \subset \Omega$. Now consider a function $\widehat{w} \in C_{0}^{2}(\Omega)$ having compact support in $\mathcal{S}^{2 \varepsilon}$ and such that $\widehat{w} \equiv w$ on $\overline{\mathcal{S}^{\varepsilon}}$. For example, a function $\widehat{w}$ with the desired properties can be constructed as follows. By the compactness of $\overline{\mathcal{S}^{\varepsilon}}$, we can find a finite number of open balls $\left\{B_{\frac{3}{2} \varepsilon}\left(x_{k}\right)\right\}_{k=1}^{N}$ such that $x_{1}, \ldots, x_{N} \in \mathcal{S}$ and

$$
\overline{\mathcal{S}^{\varepsilon}} \subset \bigcup_{k=1}^{N} B_{\frac{3}{2} \varepsilon}\left(x_{k}\right) .
$$

Now let $\left\{\psi_{k}\right\}_{k=1}^{N}$ be a smooth partition of unity on $\overline{\mathcal{S}^{\varepsilon}}$ subordinate to the open covering $\left\{B_{\frac{3}{2} \varepsilon}\left(x_{k}\right)\right\}_{k=1}^{N}$. Then we can define

$$
\widehat{w}(x)= \begin{cases}\sum_{k=1}^{N} \psi_{k}(x) w(x) & \text { for } x \in \mathcal{S}^{2 \varepsilon} \\ 0 & \text { otherwise. }\end{cases}
$$

We are now going to construct the function $\widetilde{w}$ on $\Omega \backslash \overline{\mathcal{S}^{\varepsilon}}$ with $\varepsilon>0$ small enough so that (2.4) holds. As noted in (2.5), one has that $\overline{\mathcal{S}^{\varepsilon}} \subset \Omega$ is connected. For such $\varepsilon$ one also has that $\partial\left(\Omega \backslash \overline{\mathcal{S}^{\varepsilon}}\right)=\partial \Omega \cup \partial \mathcal{S}^{\varepsilon}$ with $\partial \Omega \cap \partial \mathcal{S}^{\varepsilon}=\emptyset$.

Let $\theta>0$ be as in Step II of the proof and let $\nu_{\varepsilon}=\nu_{\varepsilon}(x)$ denote the outward unit normal to $\partial \mathcal{S}_{\varepsilon}$ at the point $x$. In the bounded open domain $\mathcal{U}:=\Omega \backslash \overline{\mathcal{S}^{\varepsilon}}$ with $C^{1,1}$ boundary $\partial \Omega \cup \partial \mathcal{S}^{\varepsilon}$, consider the weak solution $v \in H^{1}(\mathcal{U})$ of the uniformly elliptic boundary value problem

$$
\left\{\begin{array}{l}
\operatorname{div}(A(x) \nabla v)=-\operatorname{div}(A(x) \nabla \widehat{w})+\theta:=f \quad \text { in } \mathcal{U}, \\
\left\langle\nu_{\varepsilon}, A \nabla v\right\rangle=0 \quad \text { on } \partial \mathcal{S}_{\varepsilon}, \\
v=0 \quad \text { on } \partial \Omega .
\end{array}\right.
$$

We note that all of the connected components of $\partial \mathcal{U}$ are compact and separated by a positive distance, which aids the regularity analysis of $v$. Since $\widehat{w} \in C_{0}^{2}(\Omega)$ and the coefficients of $A$ are $C^{1}(\Omega)$, one has $f \in C(\bar{\Omega})$. Denoting by $H_{\partial \Omega}^{1}(\mathcal{U})$ the subspace of $H^{1}(\mathcal{U})$ whose elements have zero trace on the component $\partial \Omega$ and using $f \in L^{2}(\mathcal{U})$, one knows that there exists a unique $v \in H_{\partial \Omega}^{1}(\mathcal{U})$ which solves (3.26) in the sense that

$$
\mathcal{B}(v, \psi)=(f, \psi)_{L^{2}(\mathcal{U})}, \quad \forall \psi \in H_{\partial \Omega}^{1}(\mathcal{U}),
$$

where $\mathcal{B}$ is the usual bilinear form associated to the PDE (see Proposition 8.8 of Salsa [28], for example). Since $f \in L^{p}(\mathcal{U})$ for each $p \geq 1$ and since the coefficients of $A$ are uniformly continuous on $\mathcal{U}$, harmonic analysis techniques such as Calderon-Zygmund theory, interpolation and comparison with constant coefficient operators for the uniformly elliptic PDE yields

$$
v \in W_{\mathrm{loc}}^{2, p}(\mathcal{U}) \quad \text { for each } p \in[1, \infty) \text {. }
$$


as shown for elliptic systems in Remark 15.16 and Theorem 15.18 of Ambrosio [1]. In particular, $v$ is a strong solution of the PDE and satisfies the equation pointwise almost everywhere. Moreover, since $\partial \mathcal{U}$ is at least $C^{1}$, by applying Theorem 4.1 of Stampacchia [29] with $p>n$, one has that $v$ admits a representative

$$
v^{*} \in C(\overline{\mathcal{U}}),
$$

where $v^{*}$ will vanish on the Dirichlet component $\partial \Omega$. Combining (3.27) and (3.28), one has that $v \in C(\overline{\mathcal{U}}) \cap W_{\text {loc }}^{2, p}(\mathcal{U})$ with $p>n$ is a strong solution of the Dirichlet problem

$$
\left\{\begin{array}{l}
\operatorname{div}(A(x) \nabla v)=f \quad \text { in } \mathcal{U}, \\
v=v^{*} \quad \text { on } \partial \mathcal{U}
\end{array}\right.
$$

with $\partial \mathcal{U} \in C^{1,1}$. Such a solution is unique by Corollary 9.18 of Gilbarg-Trudinger [15]. Take a sequence $\varphi_{k} \in W^{2, p}(\mathcal{U})$ which converges uniformly to $v^{*}$ on $\partial \mathcal{U}$. By Theorem 9.15 of [15] there exists a unique strong solution $u_{k} \in W^{2, p}(\mathcal{U})$ of the Dirichlet problem with $u_{k}-\varphi_{k} \in W_{0}^{1, p}(\mathcal{U})$. The $W^{2, p}$ estimate of Lemma 9.17 of [15] then shows that $u_{k}$ is a bounded sequence in $W^{2, p}(\mathcal{U})$ and hence has a weakly converging subsequence whose weak limit is a $W^{2, p}(\mathcal{U})$ strong solution, which must agree with $v$ by the uniqueness. Since $p>n$, we also have $v \in C^{1}(\overline{\mathcal{U}})$.

Now we define $\widetilde{w}:=v+\widehat{w}$ on $\mathcal{U}=\Omega \backslash \overline{\mathcal{S}^{\varepsilon}}$, so that we immediately have $\widetilde{w} \in C^{1}(\overline{\mathcal{U}}) \cap W^{2, q}(\mathcal{U})$ for some $q>n$. By the regularity of the matrix of the coefficients $A(x)$ and of the function $\widetilde{w}$, we easily see that $\widetilde{w}$ satisfies condition $(2.10)$ for some $K>0$. Moreover, since $v \in W^{2, q}(\mathcal{U})$ is a strong solution of (3.26), for a.e. $x \in \mathcal{U}$ we have

$$
\operatorname{div}(A(x) \nabla \widetilde{w})=\operatorname{div}(A(x) \nabla v)+\operatorname{div}(A(x) \nabla \widehat{w})=\theta>0,
$$

which is condition (2.9). Finally, since $w=\widehat{w}$ on $\overline{\mathcal{S}^{\varepsilon}}$, we have

$$
\nabla w-\nabla \widetilde{w}=\nabla \widehat{w}-\nabla \widetilde{w}=-\nabla v \quad \text { on } \partial \mathcal{S}^{\varepsilon} .
$$

Thus by (3.26) we see that

$$
\left\langle A(x)(\nabla w-\nabla \widetilde{w}), \nu_{\varepsilon}\right\rangle=-\left\langle A(x) \nabla v, \nu_{\varepsilon}\right\rangle=0 \quad \text { on } \partial \mathcal{S}^{\varepsilon} .
$$

This concludes the proof of Step III, and hence also the proof of Theorem 3.1.

\section{Sobolev spaces with matrix valued weights}

In this section, we present the functional setting in which we will study questions of existence and uniqueness of weak solutions to the Dirichlet and Cauchy-Dirichlet problem for degenerate elliptic (1.3) and degenerate parabolic and hyperbolic equations (1.4) and (1.5). This involves studying first order Sobolev spaces with matrix valued weights $A$ which are calibrated for use in the applications and encode the degeneracy in terms of the set $\mathcal{S}$. We will make minimal assumptions on $A$ and $\mathcal{S}$ in order to recover most of the useful properties that are present in the situation without weights, which will correspond to choosing $A$ as the identity matrix everywhere on $\Omega$ (or more generally as a uniformly positive definite matrix valued function). More precisely, we will assume that

$$
\left\{\begin{array}{l}
(i) \quad \text { the Poincaré inequality }(3.1) \text { holds; } \\
\text { (ii) } \quad A \equiv\left[a_{i j}\right], \text { with } a_{i j}=a_{j i} \in C^{0}(\bar{\Omega}) \text {, is nonnegative definite, } \\
\text { (iii) } \quad \text { the degeneracy set } \mathcal{S} \text { (which is closed) has zero Lebesgue measure. }
\end{array}\right.
$$

We note that we have made milder regularity assumptions on $A$ (condition (ii) of (4.1)) and milder structural assumptions on $\mathcal{S}$ (condition (iii) of (4.1)) with respect to (2.1) and 
(2.3). On the other hand, we assume here the validity of the Poincaré inequality, which follows by Theorem 3.1 in the presence of the stronger regularity and structural assumptions. Hence the class of weights $A$ defined by (2.1) and (2.3) enters into this theory, as will other classes such as those for which $A$ has a uniformly elliptic direction (introduced in [20]), which also ensures the validity of the Poincaré inequality (see Theorem 2.1 of [20]). We will not prove most of the results of this section, since they follow from the same arguments employed in [20], which treated only the Hilbert case of $L^{2}$-based spaces. In particular, the arguments really depend on mild regularity and structural conditions on $A$ and $\mathcal{S}$ provided that one has the Poincaré inequality.

We begin with the definition of the spaces, which will at first employ the notion of strong derivatives; that is, we will define the spaces as completions of regular functions with respect to the natural norm involving $A$. The question of the equivalence with respect to using some notion of weak derivative to define the Sobolev spaces as natural subspaces of Lebesgue spaces will be discussed later, as will the question of suitable representation formulas which allow one to perform standard calculus on suitable representatives with weak a priori regularity (see the discussion beginning with Theorem 4.3 below).

Definition 4.1 Assume that condition (4.1) holds and let $1 \leq p<\infty$.

(a) Let $V^{p}(\Omega ; A)$ be the set of functions $u \in C^{1}(\Omega)$ such that

$$
\int_{\Omega}|u|^{p} d x+\int_{\Omega}\langle A(x) \nabla u, \nabla u\rangle^{p / 2} d x<\infty
$$

and define $W^{1, p}(\Omega ; A)$ as the completion of $V^{p}(\Omega ; A)$ with respect to the norm

$$
\begin{aligned}
\|u\|_{W^{1, p}(\Omega ; A)} & :=\left(\int_{\Omega}|u|^{p} d x+\int_{\Omega}\langle A(x) \nabla u, \nabla u\rangle^{p / 2} d x\right)^{1 / p} \\
& =\left(\|u\|_{L^{p}(\Omega)}^{p}+\||\sqrt{A} \nabla u|\|_{L^{p}(\Omega)}^{p}\right)^{1 / p}
\end{aligned}
$$

(b) Define $W_{0}^{1, p}(\Omega ; A)$ as the completion of $C_{0}^{1}(\Omega)$ with respect to the norm

$$
\|u\|_{W_{0}^{1, p}(\Omega ; A)}:=\||\sqrt{A} \nabla u|\|_{L^{p}(\Omega)}
$$

The spaces $W^{1, p}(\Omega ; A), W_{0}^{1, p}(\Omega ; A)$ are Banach spaces for each $p$. For $p=2$, the spaces are Hilbert spaces, which will be denoted by $H^{1}(\Omega ; A)$ and $H_{0}^{1}(\Omega ; A)$ and whose norms (4.3) and (4.4) are induced by the inner products

$$
\langle u, v\rangle_{H^{1}(\Omega ; A)}:=\int_{\Omega} u v d x+\int_{\Omega}\langle A(x) \nabla u, \nabla v\rangle d x \quad\left(u, v \in V^{2}(\Omega ; A)\right),
$$

and

$$
\langle u, v\rangle_{H_{0}^{1}(\Omega ; A)}:=\int_{\Omega}\langle A(x) \nabla u, \nabla v\rangle d x \quad\left(u, v \in C_{0}^{1}(\Omega)\right)
$$

respectively.

A first application of the Poincaré inequality are the following expected facts about equivalent norms and continuous immersions (see Proposition 3.3 of [20] for the proof in the case $p=2$ which carries over without change here). We will denote by

$$
\mathcal{W}_{p}=\left\{w \in C^{1}(\Omega): \int_{\Omega}|w|^{p} d x<\infty\right\}
$$

Proposition 4.2 Assume that condition (4.1) holds and let $C_{\mathcal{P}}$ be the Poincaré constant in (3.1). Then 
(a) $W_{0}^{1, p}(\Omega ; A)$ is a subspace of $W^{1, p}(\Omega ; A)$ and one has the following equivalence of norms

$$
\left(C_{\mathcal{P}}^{p}+1\right)^{-1 / p}\|u\|_{W^{1, p}(\Omega ; A)} \leq\|u\|_{W_{0}^{1, p}(\Omega ; A)} \leq\|u\|_{W^{1, p}(\Omega ; A)},
$$

for any $u \in W_{0}^{1, p}(\Omega ; A)$.

(b) The spaces $W^{1, p}(\Omega ; A), W_{0}^{1, p}(\Omega ; A)$ are continuously immersed into $L^{p}(\Omega)$; that is, any Cauchy sequence in $\left(V^{p}(\Omega ; A),\|\cdot\|_{W^{1, p}(\Omega ; A)}\right),\left(C_{0}^{1}(\Omega),\|\cdot\|_{W_{0}^{1, p}(\Omega ; A)}\right)$ is a Cauchy sequence in $\left(\mathcal{W}_{p}(\Omega),\|\cdot\|_{L^{p}(\Omega)}\right)$ and these inclusions pass to the relevant quotient spaces to give well defined linear maps $i, i_{0}$ such that

$$
\|i(u)\|_{L^{p}(\Omega)} \leq\|u\|_{W^{1, p}(\Omega ; A)} \quad \text { for any } u \in W^{1, p}(\Omega ; A),
$$

and

$$
\left\|i_{0}(u)\right\|_{L^{p}(\Omega)} \leq C_{\mathcal{P}}\|u\|_{W_{0}^{1, p}(\Omega ; A)} \quad \text { for any } u \in W_{0}^{1, p}(\Omega ; A) .
$$

We now address the question of selecting suitable representatives $u$ from the space of equivalence classes in $W^{1, p}(\Omega ; A)$ and $W_{0}^{1, p}(\Omega ; A)$. The starting point is the following generalization of Theorem 3.5 of [20], which treats the case $p=2$, and again the proof trivially adapts to general $p$ in the presence of the Poincaré inequality.

Theorem 4.3 Let assumption (4.1) be satisfied. Then for every $\left[\left\{u_{n}\right\}\right] \in W^{1, p}(\Omega ; A)$ there exist unique elements $u \in L^{p}(\Omega), U \in\left[L^{p}(\Omega)\right]^{N}$ such that

(a) $\left\|u_{n}-u\right\|_{L^{p}(\Omega)} \rightarrow 0,\left\|\sqrt{A} \nabla u_{n}-U\right\|_{L^{p}(\Omega)} \rightarrow 0$ as $n \rightarrow \infty$;

(b) $[\sqrt{A}]^{-1} U$ is the weak gradient of $u$ in the open set $\Omega \backslash \mathcal{S}$; that is, the gradient in the sense of distributions of $u \in L_{\mathrm{loc}}^{1}(\Omega)$ is represented by $[\sqrt{A}]^{-1} U \in\left[L_{\mathrm{loc}}^{1}(\Omega \backslash \mathcal{S})\right]^{N}$ when the test functions have support in the open set $\Omega \backslash \mathcal{S}$;

(c) if $\left|[\sqrt{A}]^{-1}\right| \in L^{p^{\prime}}(\Omega)$, then $[\sqrt{A}]^{-1} U$ is the weak gradient of $u$ in all of $\Omega$;

(d) $\left\|\left[\left\{u_{n}\right\}\right]\right\|_{W^{1, p}(\Omega ; A)}^{p}=\|u\|_{L^{p}(\Omega)}^{p}+\||U|\|_{L^{p}(\Omega)}^{p}$.

Using Theorem 4.3, we have the following embedding result which generalizes Corollary 3.6 of $[20]$.

Corollary 4.4 Under the hypotheses of Theorem 4.3, if $\left[\left\{u_{n}\right\}\right] \in W^{1, p}(\Omega ; A)$ has its $L^{p}(\Omega)$ class $i(u)=0$, then $U=0 \in\left[L^{p}(\Omega)\right]^{N}$ as well. In particular, the immersions $i, i_{0}$ of Proposition 4.2 are embeddings.

The injectivity of the maps $i, i_{0}$ allows us to identify $W^{1, p}(\Omega ; A)$ and $W_{0}^{1, p}(\Omega ; A)$ with subsets of $L^{p}(\Omega)$ for each $p \in[1, \infty)$. In particular, this shows that $W^{1, p}(\Omega ; A)$ and $W_{0}^{1, p}(\Omega ; A)$ are separable for each $p \in[1, \infty]$. We note that from now on we will use the slight abuse of notation by using the same symbol $u$ for an element $\left[\left\{u_{n}\right\}\right] \in W^{1, p}(\Omega ; A), W_{0}^{1, p}(\Omega ; A)$ as well as for the $L^{p}(\Omega)$ class that it determines in accordance with the Representation Theorem 4.3.

As pointed out in [20], while $[\sqrt{A}]^{-1} U$ gives an $L_{\text {loc }}^{1}$ weak gradient away from the singular set $\mathcal{S}$, such a weak gradient may well not exist everywhere on $\Omega$ if the integrability condition $\left|[\sqrt{A}]^{-1}\right| \in L^{p^{\prime}}(\Omega)$ in part (iii) of Theorem 4.3 fails (see Example 3.9 of [20] for a counterexample with $p=2$ ). However, as done in [20], given the properties of the function $U \in\left[L^{p}(\Omega)\right]^{N}$ in the theorem, the following notion helps to clarify the situation.

Definition 4.5 For each $u \in W^{1, p}(\Omega ; A)$, we define its quasi-gradient $\nabla^{*} u$ by

$$
\nabla^{*} u:=[\sqrt{A}]^{-1} U,
$$

which is a measurable vector valued function defined almost everywhere in $\Omega$; that is, away from the singular set $\mathcal{S}$ of $A$. 
Remark 4.6 This quasi-gradient satisfies the following properties:

(a) if $u \in W^{1, p}(\Omega ; A)$ admits first order derivatives in $L_{l o c}^{1}(\Omega)$, then $\nabla^{*} u=\nabla u$ a.e. in $\Omega$;

(b) we have

$$
\|u\|_{W^{1, p}(\Omega ; A)}^{p}=\|u\|_{L^{p}(\Omega)}^{p}+\left\|\left|\sqrt{A} \nabla^{*} u\right|\right\|_{L^{p}(\Omega)}^{p}=\int_{\Omega}|u|^{p} d x+\int_{\Omega}\left\langle A(x) \nabla^{*} u, \nabla^{*} u\right\rangle^{p / 2} d x
$$

(c) we have

$$
\|u\|_{W_{0}^{1, p}(\Omega ; A)}^{p}=\left\|\left|\sqrt{A} \nabla^{*} u\right|\right\|_{L^{p}(\Omega)}^{p}=\int_{\Omega}\left\langle A(x) \nabla^{*} u, \nabla^{*} u\right\rangle^{p / 2} d x ;
$$

(d) since $\nabla^{*} u=\nabla u$ in $\Omega \backslash \mathcal{S}$, and $\mathcal{S}$ has zero Lebesgue measure, $\nabla^{*} u=0$ a.e. on each level set of the function $u$.

In order to simplify notation, we will often write $\nabla u$ in place of $\nabla^{*} u$ as they agree almost everywhere; however, one should keep in mind that $\nabla u$ may not have components which are $L_{\text {loc }}^{1}$ near the singular set $\mathcal{S}$ if the integrability condition $\left|[\sqrt{A}]^{-1}\right| \in L^{p^{\prime}}(\Omega)$ fails. In particular, in section 5 we will make constant use of the simple identities

$$
\langle A \nabla u, \nabla v\rangle=\langle\sqrt{A} \nabla u, \sqrt{A} \nabla v\rangle \quad \text { and } \quad\langle b, \nabla u\rangle=\left\langle[\sqrt{A}]^{-1} b, \sqrt{A} \nabla u\right\rangle
$$

for $u, v \in W^{1, p}(\Omega ; A)$ and $[\sqrt{A}]^{-1} b \in L^{\infty}(\Omega)$.

We now turn our attention to the problem of establishing conditions under which the norm in $W^{1, p}(\Omega ; A)$ or $W_{0}^{1, p}(\Omega ; A)$ can be calculated directly by the integral expression given by (4.3) or (4.4) as opposed to calculating a limit of such expressions along a Cauchy sequence representative. In this direction, we have the following two propositions (which are trivial generalizations of Propositions 3.10 and 3.11 of [20]).

Proposition 4.7 Assume the conditions (4.1) and let $p \in[1, \infty)$. Then the following statements hold:

(a) if $u \in W^{1, p}(\Omega)$, then $u \in W^{1, p}(\Omega ; A)$ and

$$
\|u\|_{W^{1, p}(\Omega ; A)}=\left(\int_{\Omega}|u|^{p} d x+\int_{\Omega}\langle A(x) \nabla u, \nabla u\rangle^{p / 2} d x\right)^{1 / p} ;
$$

(b) if $u \in W_{0}^{1, p}(\Omega)$, then $u \in W_{0}^{1, p}(\Omega ; A)$ and

$$
\|u\|_{W_{0}^{1, p}(\Omega ; A)}=\left(\int_{\Omega}\langle A(x) \nabla u, \nabla u\rangle^{p / 2} d x\right)^{1 / p} .
$$

Proposition 4.8 Assume the conditions (4.1) and let $p \in[1, \infty)$. If $u \in W_{l o c}^{1, p}(\Omega)$ and

$$
\int_{\Omega}|u|^{p} d x+\int_{\Omega}\langle A(x) \nabla u, \nabla u\rangle^{p / 2} d x<\infty,
$$

then $u \in W^{1, p}(\Omega ; A)$ and

$$
\|u\|_{W^{1, p}(\Omega ; A)}=\left(\int_{\Omega}|u|^{p} d x+\int_{\Omega}\langle A(x) \nabla u, \nabla u\rangle^{p / 2} d x\right)^{1 / p} .
$$

Remark 4.9 By Proposition 4.7, the space $W_{0}^{1, p}(\Omega ; A)$ coincides with the completion with respect to the norm (4.4) of the space of Lipschitz functions with compact support in $\Omega$ since such Lipschitz functions are included in $W_{0}^{1, p}(\Omega)$. Similarly, Proposition 4.8 shows that $W^{1, p}(\Omega ; A)$ could be alternatively defined as the completion with respect to the norm (4.3) of the space of locally Lipschitz functions in $\Omega$ satisfying (4.2). 
We next address the question of taking positive and negative parts, which will be useful in the formulation of maximum and comparison principles for weak solutions to the associated degenerate elliptic and parabolic PDEs in section 5. Given $u \in W^{1, p}(\Omega ; A), W_{0}^{1, p}(\Omega ; A)$, if one starts from the positive part of a Cauchy sequence defining $u$, one sees that this sequence determines an element, called $u^{+}$, in $W^{1, p}(\Omega ; A), W_{0}^{1, p}(\Omega ; A)$. This claim is the content of the next lemma (which is a trivial generalization of Theorem 3.13 of [20]).

Lemma 4.10 Assume the conditions (4.1) and let $p \in[1, \infty)$. Then the following statements hold:

(a) If $u \in W^{1, p}(\Omega ; A)$, then $u^{+}, u^{-} \in W^{1, p}(\Omega ; A)$ and one has

$$
\|u\|_{W^{1, p}(\Omega ; A)}^{p}=\left\|u^{+}\right\|_{W^{1, p}(\Omega ; A)}^{p}+\left\|u^{-}\right\|_{W^{1, p}(\Omega ; A)}^{p} ;
$$

(b) if $u \in W_{0}^{1, p}(\Omega ; A)$, then $u^{+}, u^{-} \in W_{0}^{1, p}(\Omega ; A)$;

$$
\|u\|_{W_{0}^{1, p}(\Omega ; A)}^{p}=\left\|u^{+}\right\|_{W_{0}^{1, p}(\Omega ; A)}^{p}+\left\|u^{-}\right\|_{W_{0}^{1, p}(\Omega ; A)}^{p} .
$$

Next, we note that elements of $W^{1, p}(\Omega ; A), W_{0}^{1, p}(\Omega ; A)$ can be approximated by smooth functions by using standard procedures. The following result generalizes Theorem 3.16 of [20].

Proposition 4.11 Assume the conditions (4.1) and let $p \in[1, \infty)$. Then the following statements hold:

(a) $C_{0}^{\infty}(\Omega) \subset W_{0}^{1, p}(\Omega ; A)$ is dense;

(b) $C^{\infty}(\Omega) \cap W^{1, p}(\Omega ; A) \subset W^{1, p}(\Omega ; A)$ is dense.

We remark that combining Corollary 4.4 with part (a) of Proposition 4.11 shows that $W_{0}^{1, p}(\Omega ; A)$ is densely embedded in $L^{p}(\Omega)$ for every $p \in[1, \infty)$.

Finally, we give the following characterization of elements in the dual space $H^{-1}(\Omega ; A)=$ $\left[H_{0}^{1}(\Omega ; A)\right]^{\prime}$. This dual space will play a prominent role in the applications to degenerate PDEs in the next section.

Proposition 4.12 Assume the conditions (4.1) hold with $p=2$. Given $f \in H^{-1}(\Omega ; A)$ there exists a collection $\left\{f_{k}\right\}_{k=0}^{n} \subset L^{2}(\Omega)$ such that

$$
\langle f, u\rangle=\int_{\Omega}\left[f_{0} u+\sum_{k=1}^{n} f_{k}(\sqrt{A} \nabla u)_{k}\right] d x, \quad u \in H_{0}^{1}(\Omega ; A) .
$$

Proof. The argument is classical, but we give it in order to illustrate the role of the representation formulas of Theorem 4.3. Exploiting the Poicaré inequality, equipping $H_{0}^{1}(\Omega ; A)$ with the modified inner product

$$
(u, v)_{H^{1}(\Omega ; A)}:=(u, v)_{H_{0}^{1}(\Omega ; A)}+(u, v)_{L^{2}(\Omega)}
$$

also yields a Hilbert space. For each $f \in H^{-1}(\Omega ; A)$, one applies the Riesz representation theorem with respect to the inner product (4.12) to find a unique $\varphi \in H_{0}^{1}(\Omega ; A)$ such that

$$
\langle f, u\rangle=(\varphi, u)_{H_{0}^{1}(\Omega ; A)}+(\varphi, u)_{L^{2}(\Omega)}
$$

Using Theorem 4.3, one selects representatives $u, \varphi \in L^{2}(\Omega)$ with associated quasi-gradients $\nabla u=[\sqrt{A}]^{-1} U, \nabla \varphi=[\sqrt{A}]^{-1} \Phi$ in order to find

$$
\langle f, u\rangle=\int_{\Omega}\langle\sqrt{A} \nabla \varphi, \sqrt{A} \nabla u\rangle d x+\int_{\Omega} \varphi u d x
$$


which yields (4.11) by selecting $f_{0}=\varphi$ and $f_{k}=(\sqrt{A} \nabla \varphi)_{k}$.

We conclude by noting the obvious fact that there is a natural continuous injection of $L^{2}(\Omega)$ into $H^{-1}(\Omega ; A)$ provided by $f \mapsto \ell_{f}$ with

$$
\left\langle\ell_{f}, u\right\rangle:=(f, u)_{L^{2}(\Omega)}, \quad u \in H_{0}^{1}(\Omega ; A) .
$$

The injectivity follows from the density of $C_{0}^{\infty}(\Omega)$ in $L^{2}(\Omega)$ and $H_{0}^{1}(\Omega ; A)$ while the continuity follows from the standard combination of the Cauchy-Schwarz and Poincaré inequalities

$$
\left|(f, u)_{L^{2}(\Omega)}\right| \leq\|f\|_{L^{2}(\Omega)}|| u\left\|_{L^{2}(\Omega)} \leq C_{\mathcal{P}}\right\| u\left\|_{H_{0}^{1}(\Omega ; A)}|| f\right\|_{L^{2}(\Omega)} .
$$

\section{Applications to degenerate PDEs}

In this section, we put the Poincare inequality of Theorem 3.1 to work in the context of the Sobolev spaces with matrix valued weights $A$ and domains $\Omega$ satisfying (2.1) and (2.3) to treat questions of existence, uniqueness and qualitative properties of solutions of degenerate PDEs. Assuming that (2.1) and (2.3) hold, we also have the rest of the conditions in (4.1) needed to develop all of the results on the Sobolev spaces with matrix valued weights presented in section 4. Since the applications make use of standard techniques, we will be brief. However, we will be precise with respect to the sense of solutions, the needed assumptions of the coefficients and quantitative role of the Poincaré constant.

As is well known, the validity of a Poincaré inequality calibrated to the principal part of the differential operator $L$ acting in the spatial derivatives yields a common framework in which to operate. This framework involves a Hilbert triple

$$
V \hookrightarrow H \hookrightarrow V^{\prime}
$$

where $V$ and $H$ are separable Hilbert spaces, $V^{\prime}$ is the dual space to $V$, the injections are continuous and the image of $V$ is dense in $H$, whose dual is identified with $H$. Setting

$$
V=H_{0}^{1}(\Omega ; A), \quad H=L^{2}(\Omega), \text { and } V^{\prime}=H^{-1}(\Omega ; A),
$$

the results of section 4 show that (5.16) does indeed give a Hilbert triple.

For the degenerate elliptic equations, solutions to the Dirichlet problem will be found in the Sobolev space $V$, while for the evolution equations the solutions will be found as suitable elements $u=u(t)$ belonging to Sobolev spaces of $V$-valued maps depending on the real time parameter $t$. To this end, we recall that for a separable Hilbert space $X$ and $T \in(0,+\infty)$, one defines the Hilbert space $L^{2}(0, T ; X)$ as the equivalence classes of maps $u$ where

$$
t \in(0, T) \mapsto u(t) \in X \quad \text { is defined almost everywhere and is measurable }
$$

and

$$
\|u\|_{L^{2}(0, T ; X)}:=\left(\int_{0}^{T}\|u(t)\|_{X}^{2} d t\right)^{1 / 2}<+\infty .
$$

For the parabolic problems, the solution will be a map $u \in L^{2}(0, T ; V) \hookrightarrow L^{2}\left(0, T ; V^{\prime}\right)$ whose derivative in $t$ as $V^{\prime}$-valued distribution admits a representative in $L^{2}\left(0, T ; V^{\prime}\right)$, which will be denoted by $\frac{d u}{d t}$. For the hyperbolic problems, the solutions will be maps $u \in L^{2}(0, T ; V) \hookrightarrow$ $L^{2}(0, T ; H) \hookrightarrow L^{2}\left(0, T ; V^{\prime}\right)$ such that

$$
\frac{d u}{d t} \in L^{2}(0, T ; H) \quad \text { and } \quad \frac{d^{2} u}{d t^{2}} \in L^{2}\left(0, T ; V^{\prime}\right) .
$$

In this setting, under suitable assumptions on the coefficients of $L$ the evolution PDEs can be interpreted as equations in $L^{2}\left(0, T ; V^{\prime}\right)$ which then reduce to ODEs for real-valued distributions $\mathcal{D}^{\prime}((0, T))$ when tested on arbitrary elements $v \in V$. Various well known properties of such time dependent Sobolev spaces will be used, such as those contained in sections 18.1 and 18.3 of Dautray-Lions [6]. 


\subsection{Degenerate elliptic problems}

We begin with the Dirichlet problem associated to the equation (1.3); that is,

$$
\begin{cases}L u=f & \text { in } \Omega \\ u=0 & \text { on } \partial \Omega,\end{cases}
$$

where $L$ has the form $L u:=\operatorname{div}\{A \nabla u\}+\langle b, \nabla u\rangle+c u$ and $f \in H^{-1}(\Omega ; A)$ is given. If $A$ satisfies the conditions $(4.1)$ and if $(b, c)$ satisfy mild assumptions, the following notion of weak solution makes sense.

Definition 5.1 Let $f \in H^{-1}(\Omega ; A)$. We say $u \in H_{0}^{1}(\Omega ; A)$ is a weak solution of (5.19) provided that

$$
\mathcal{B}(u, v)=-\langle f, v\rangle \quad \text { for every } v \in H_{0}^{1}(\Omega ; A),
$$

where

$$
\mathcal{B}(u, v):=\int_{\Omega}\{\langle A(x) \nabla u, \nabla v\rangle-\langle b(x), \nabla u\rangle v-c(x) u v\} d x
$$

is the natural bilinear form associated to $L$ and $\nabla u, \nabla v$ are the quasi-gradients of $u, v$ respectively. If $\mathcal{B}$ is continuous, then it is equivalent to require the validity of (5.20) for each $v \in C_{0}^{1}(\Omega)$.

The Lax-Milgram theorem yields the existence of a unique weak solution provided that the bilinear form $\mathcal{B}$ is bounded and coercive on $H_{0}^{1}(\Omega ; A) \times H_{0}^{1}(\Omega ; A)$; that is, if there exist $\alpha, \beta>0$ such that

$$
|\mathcal{B}(u, v)| \leq \alpha\|u\|_{H_{0}^{1}(\Omega ; A)}\|v\|_{H_{0}^{1}(\Omega ; A)}, \quad u, v \in H_{0}^{1}(\Omega ; A)
$$

and

$$
\mathcal{B}(u, u) \geq \beta\|u\|_{H_{0}^{1}(\Omega ; A)}^{2}, \quad u \in H_{0}^{1}(\Omega ; A) .
$$

Making use of the simple identities (4.10) and estimating from above in the standard way, one finds that (5.22) holds with

$$
\alpha=1+C_{\mathcal{P}} \|\left[[\sqrt{A}]^{-1} b\left\|_{L^{\infty}(\Omega)}+C_{\mathcal{P}}^{2}\right\| c \|_{L^{\infty}(\Omega)}<+\infty\right.
$$

provided that

$$
[\sqrt{A}]^{-1} b \in L^{\infty}(\Omega) \quad \text { and } \quad c \in L^{\infty}(\Omega) .
$$

We note that when $A$ is positive definite and the equation is elliptic, the first condition in (5.24) is the familiar condition $b \in L^{\infty}(\Omega)$.

On the other hand, estimating the quadratic form from below by way of the CauchySchwarz inequality, one obtains (5.23) provided that (2.1), (2.3) and (5.24) hold with

$$
\beta:=1-C_{\mathcal{P}}\left\|\left|[\sqrt{A}]^{-1} b\right|\right\|_{L^{\infty}(\Omega)}-C_{\mathcal{P}}^{2}\left\|c^{+}\right\|_{L^{\infty}(\Omega)}>0,
$$

where $C_{\mathcal{P}}$ is the Poincaré constant. Summing up, one has the following existence theorem.

Theorem 5.2 Let assumptions (2.1), (2.3) and (5.24) be satisfied and suppose that the relation (5.25) holds. Then for each $f \in H^{-1}(\Omega ; A)$ there exists a unique solution $u \in H_{0}^{1}(\Omega ; A)$ to the Dirichlet problem (5.19). Moreover, the unique solution u satisfies the estimate

$$
\|u\|_{H_{0}^{1}(\Omega ; A)} \leq \frac{1}{\beta}\|f\|_{H^{-1}(\Omega: A)} .
$$


A variant of the above proof of the coercivity estimate, which uses Young's inequality instead of the Poincaré inequality in order to estimate the first order terms of the quadratic form $\mathcal{B}(u, u)$, shows that if

$$
\sup _{\Omega} c<-\frac{1}{4}\left\|\mid[\sqrt{A}]^{-1} b\right\|_{\infty}^{2}
$$

then (5.23) holds with

$$
\beta:=1+\frac{\left\|\left|[\sqrt{A}]^{-1} b\right|\right\|_{\infty}^{2}}{4 \sup _{\Omega} c} \in(0,1) .
$$

This yields the following existence theorem, in the case when $c$ is negative and large enough on the domain $\Omega$.

Theorem 5.3 Let assumptions (2.1), (2.3) and (5.24) be satisfied and suppose that the relation (5.27) holds. Then for each $f \in H^{-1}(\Omega ; A)$ there exists a unique solution $u \in H_{0}^{1}(\Omega ; A)$ to the Dirichlet problem (5.19). Moreover, the unique solution u satisfies the estimate (5.26), with $\beta$ defined as in (5.28).

Remark 5.4 From Theorems 5.2 and 5.3 and from (5.26) it follows that the solution map, that associates to each $f \in H^{-1}(\Omega ; A)$ the unique solution $u \in H_{0}^{1}(\Omega ; A)$ to problem (5.19) is continuous and linear. Moreover, this solution operator is the inverse of the bounded linear operator $L: u \in H_{0}^{1}(\Omega ; A) \mapsto f:=L u \in H^{-1}(\Omega ; A)$ defined by

$$
\langle L u, v\rangle:=\mathcal{B}(u, v), \quad u, v \in H_{0}^{1}(\Omega ; A),
$$

and hence $L$ establishes an isomorphism between $H_{0}^{1}(\Omega ; A)$ and $H^{-1}(\Omega ; A)$ if the coefficients of L satisfy (2.1), (2.3), (5.24) and either (5.25) or (5.27).

We turn our attention to the validity of a weak comparison and a weak maximum principles for the degenerate elliptic equation $L u=f$ which are compatible with the weak well posedness of the Dirichlet problem (5.19). To this end, we note that the associated bilinear form $\mathcal{B}$ (with the expression (5.21)) is well defined and continuous on the larger spaces $H^{1}(\Omega ; A) \times H_{0}^{1}(\Omega ; A)$ and $H^{1}(\Omega ; A) \times H^{1}(\Omega ; A)$ if the conditions (2.1), (2.3) and (5.24) hold.

Definition 5.5 Let $A$ and $\Omega$ satisfy the assumptions of Theorem 5.2 and let $f \in H^{-1}(\Omega ; A)$. We say that

(a) $u \in H^{1}(\Omega ; A)$ is a weak subsolution of the equation $L u=f$ if

$$
\mathcal{B}(u, v)+\langle f, v\rangle \leq 0 \quad \text { for any } v \in H_{0}^{1}(\Omega ; A), v \geq 0 ;
$$

(b) $u \in H^{1}(\Omega ; A)$ is a weak supersolution to the equation $L u=f$ if

$$
\mathcal{B}(u, v)+\langle f, v\rangle \geq 0 \quad \text { for any } v \in H_{0}^{1}(\Omega ; A), v \geq 0 .
$$

By the continuity of $\mathcal{B}$, one may replace the test function space $H_{0}^{1}(\Omega ; A)$ with $C_{0}^{1}(\Omega)$.

The standard argument of using the coercivity of $\mathcal{B}$ and the choice of $v=u^{+}$in Definition 5.5 (a) yields the following weak comparison principle, where we recall that for $u \in H^{1}(\Omega ; A)$ the condition $u \leq 0$ on $\partial \Omega$ just means $u^{+} \in H_{0}^{1}(\Omega ; A)$.

Theorem 5.6 Let the assumptions of Theorem 5.2 be satisfied. Let $u \in H^{1}(\Omega ; A)$ be a subsolution to the equation $L u=0$ such that $u \leq 0$ on $\partial \Omega$. Then

$$
u \leq 0 \quad \text { a.e. in } \Omega \text {. }
$$

By linearity, one has the full comparison principle that $u \leq v$ on $\partial \Omega$ implies $u \leq v$ a.e. in $\Omega$, provided that $L u \leq L v$ in the weak sense. The following weak maximum principle follows from the standard argument. 
Corollary 5.7 Let the assumptions of Theorem 5.2 be satisfied. Suppose that $c \leq 0$ a.e. in $\Omega$. Let $u \in H^{1}(\Omega ; A)$ be a subsolution to the equation $L u=0$. Then

$$
u \leq \sup _{\partial \Omega} u^{+} \text {a.e. in } \Omega \text {. }
$$

\subsection{Degenerate parabolic problems}

Next we consider the Cauchy-Dirichlet problem associated to equation (1.4); that is

$$
\begin{cases}\partial_{t} u=L u+f(x, t) & \text { in } Q_{T}:=\Omega \times(0, T] \\ u=0 & \text { in } \partial \Omega \times(0, T], \\ u=u_{0} & \text { in } \Omega \times\{0\},\end{cases}
$$

where $L$ has the form $L u:=\operatorname{div}\{M(x, t) \nabla u\}+\langle b(x, t), \nabla u\rangle+c(x, t) u, f \in L^{2}\left(0, T ; H^{-1}(\Omega ; A)\right)$ and $u_{0} \in L^{2}(\Omega)$. As noted at the beginning of this section, we will regard $u=u(t)$ as an $H_{0}^{1}(\Omega ; A)$-valued distribution of $t \in(0, T)$. Under suitable assumptions on the coefficients $(M, b, c)$ the following notion of weak solution makes sense as detailed in section 18.3 of [6] and as sketched in Remark 5.9 below.

Definition 5.8 Given $f \in L^{2}\left(0, T ; H^{-1}(\Omega ; A)\right)$ and $u_{0} \in L^{2}(\Omega)$, we say that $u$ is a weak solution of (5.29) provided:

$$
\begin{gathered}
u \in L^{2}\left(0, T ; H_{0}^{1}(\Omega ; A)\right) \quad \text { and } \quad \frac{d u}{d t} \in L^{2}\left(0, T ; H^{-1}(\Omega ; A)\right) \\
\frac{d}{d t}\langle u(\cdot), v\rangle+\mathcal{B}(\cdot ; u(\cdot), v)=\langle f(\cdot), v\rangle \quad \text { in } \mathcal{D}^{\prime}((0, T)), \forall v \in H_{0}^{1}(\Omega ; A)
\end{gathered}
$$

and

$$
u(0)=u_{0},
$$

where

$$
\mathcal{B}(t, u, v):=\int_{\Omega}\{\langle M(x, t) \nabla u, \nabla v\rangle-\langle b(x, t), \nabla u\rangle v-c(x, t) u v\} d x \quad u, v \in H_{0}^{1}(\Omega ; A) .
$$

is the natural family of $t$-dependent bilinear forms and $\nabla u, \nabla v$ are the quasi-gradients of $u, v$.

We record some well known considerations regarding the choice of the solution space encoded by (5.30) with respect to Definition 5.8. This solution space is often denoted by $W\left(0, T ; V, V^{\prime}\right)$ with $\left(V, H, V^{\prime}\right)$ the Hilbert triple (5.16)

Remark 5.9 Let u satisfy condition (5.30) in Definition 5.8.

(a) $W\left(0, T ; V, V^{\prime}\right)$ is embedded in $C([0, T] ; H)$; that is, the function u admits a representative in $C\left([0, T] ; L^{2}(\Omega)\right.$ ) (see Theorem 1 in section 18.1 of $\left.[6]\right)$. Hence the initial condition (5.32) means $u_{0}=\lim _{t \rightarrow 0^{+}} u(t)$ in $L^{2}(\Omega)$.

(b) If the map $t \in[0, T] \rightarrow \mathcal{B}(t, \cdot, \cdot)$ taking values in bounded linear forms on $V \times V$ is essentially bounded, then the condition (5.31) is equivalent to

$$
\left\langle\frac{d u}{d t}(t), v\right\rangle+\mathcal{B}(t, u(t), v)=\langle f(t), v\rangle \text { for a.e. } t \in(0, T), \text { for every } v \in C_{0}^{1}(\Omega) .
$$

Indeed, by Proposition 7 of [6] the first term in (5.31) agrees with $\left\langle\frac{d u}{d t}(\cdot), v\right\rangle$ in $\mathcal{D}^{\prime}((0, T))$ for each $v \in V=H_{0}^{1}(\Omega ; A)$, but the continuity on $V$ of $\left\langle\frac{d u}{d t}(t), \cdot\right\rangle$ and $\langle f(t), \cdot\rangle$ for almost every $t$ means that one need only check that (5.31) holds for each $v \in C_{0}^{1}(\Omega)$, which is dense in $V$. 
(c) If we denote by $L u(t)$ as the $V^{\prime}$-valued distribution on $(0, T)$ defined by

$$
\langle L u(t), v\rangle:=\mathcal{B}(t, u(t), v), \quad v \in V=H_{0}^{1}(\Omega ; A),
$$

then (5.34) yields

$$
\frac{d u}{d t}+L u=f \quad \text { in } L^{2}\left(0, T ; H^{-1}(\Omega ; A)\right)
$$

since all of the vector valued distributions in (5.35) are representable as $L_{\mathrm{loc}}^{1}$ functions in $t$ and one can apply Proposition 5 of section 18.1 of [6].

Having transformed the problem (5.29) into the initial value problem (5.31)-(5.32) for $u(\cdot)$ satisfying (5.30), one can exploit an abstract existence and uniqueness theorem in the Hilbert triple (5.16) by using the so-called parabolic counterpart of the Lax-Milgram theorem, due to J-L. Lions (see the Comments on Chapter 10 of [3]). One needs only to verify that the family of bilinear forms $\mathcal{B}(t, \cdot, \cdot)$ with $t \in[0, T]$ is measurable in $t$, uniformly bounded and uniformly weakly coercive; that is, one needs only

$$
\forall u, v \in H_{0}^{1}(\Omega ; A), \quad t \mapsto \mathcal{B}(t, u, v) \text { defines a measurable function on }[0, T]
$$

and the existence of positive constants $\alpha, \beta, \gamma$ such that

$$
|\mathcal{B}(t, u, v)| \leq\left.\alpha|| u\right|_{H_{0}^{1}(\Omega ; A)}|| v \|_{H_{0}^{1}(\Omega ; A)}, \forall t \in[0, T], \forall u, v \in H_{0}^{1}(\Omega ; A),
$$

and

$$
|\mathcal{B}(t, u, u)| \geq \beta|| u\left\|_{H_{0}^{1}(\Omega ; A)}^{2}-\gamma\right\| u \|_{L^{2}(\Omega)}^{2}, \forall t \in[0, T], \forall u \in H_{0}^{1}(\Omega ; A) .
$$

Under the assumptions (5.36)-(5.38) there exists a unique solution of problem (5.29) in the sense of Definition 5.8 (see Theorems 1 and 2 of section 18.3 in [6]). The uniqueness follows easily from the weak coercivity assumption (5.38) and the existence in obtained using a Galerkin approximation in the separable Hilbert space $V=H_{0}^{1}(\Omega ; A)$.

The needed regularity, boundedness and coercivity will follow by making suitable hypotheses on the coefficients $(M, b, c)$ of the degenerate elliptic operator $L$. The following theorem does precisely that, where we recall that $Q_{T}=\Omega \times(0, T]$ and $\operatorname{Sym}_{n}^{+}(\mathbb{R})$ is the space of symmetric nonnegative definite matrices with real entries.

Theorem 5.10 Let $A$ and $\Omega$ satisfy the regularity and structural conditions (2.1) and (2.3). Assume that the coefficients $(M, b, c)$ of $L$ satisfy

$$
\begin{gathered}
M \in C\left(\overline{Q_{T}}, \operatorname{Sym}_{n}^{+}(\mathbb{R})\right), \\
{[\sqrt{M}]^{-1} b \in L^{\infty}\left(Q_{T}, \mathbb{R}^{n}\right) \text { and } c \in L^{\infty}\left(Q_{T}, \mathbb{R}\right)}
\end{gathered}
$$

and there exist positive constants $\mu_{1}, \mu_{2}$ such that

$$
\mu_{1} A(x) \leq M(x, t) \leq \mu_{2} A(x), \quad \forall t \in(0, T), \forall x \in \Omega,
$$

with the inequalities in the sense of quadratic forms. Then there exists a unique weak solution to the Cauchy-Dirichlet problem (5.29) in the sense of Definition 5.8

Proof. Applying the abstract existence theorem, it suffices to check that one has the needed properties (5.36)-(5.38). For the uniform upper bound (5.37) one uses the Cauchy-Schwarz inequality and the conditions (5.40) to find

$$
\begin{aligned}
|\mathcal{B}(t, u, v)| \leq & \||\sqrt{M}(t) \nabla u|\|_{L^{2}(\Omega)}\||\sqrt{M}(t) \nabla v|\|_{L^{2}(\Omega)}+C_{1}\||\sqrt{M}(t) \nabla u|\|_{L^{2}(\Omega)}\|v\|_{L^{2}(\Omega)} \\
& +C_{2}\|u\|_{L^{2}(\Omega)}\|v\|_{L^{2}(\Omega)}
\end{aligned}
$$


with $C_{1}=\left\|\left|[\sqrt{M}]^{-1} b\right|\right\|_{L^{\infty}\left(Q_{T}\right)}$ and $C_{2}=\|c\|_{L^{\infty}\left(Q_{T}\right)}$, where the lower bound in (5.41) justifies the existence of $\sqrt{M} \in C\left(\overline{Q_{T}}, \operatorname{Sym}_{n}^{+}(\mathbb{R})\right)$. Using the upper bound in (5.41) and the Poincaré inequality one obtains (5.37) with $\alpha=\alpha\left(C_{1}, C_{2}, \mu_{2}, C_{\mathcal{P}}\right)$ independent of $(t, u, v)$.

In particular, for each $t$ fixed $\mathcal{B}(t, \cdot, \cdot)$ is a bounded bilinear form on $H_{0}^{1}(\Omega ; A)$. Moreover, fixing representatives $u, v$ and their quasi-gradients in accordance with Theorem 4.3, one sees that the integrand in (5.33) is a measurable function of $t$ taking values in $L^{1}(\Omega)$ and the property (5.36) follows.

For the weak coercivity (5.38), one again estimates from below as in the classical Gårding inequality, where the lower bound in (5.41) is used. In fact, for each $u \in H_{0}^{1}(\Omega ; A)$ one finds

$$
\begin{aligned}
\mu_{1}\|u\|_{H_{0}^{1}(\Omega ; A)}^{2} & \leq \mathcal{B}(t, u, u)+\int_{\Omega}\left\{\left\langle[\sqrt{M}]^{-1} b, \sqrt{M} \nabla u\right\rangle+c u^{2}\right\} d x \\
& \leq \mathcal{B}(t, u, u)+\mu_{2}\left\|\left|[\sqrt{M}]^{-1} b\right|\right\|_{L^{\infty}\left(Q_{T}\right)}\|u\|_{H_{0}^{1}(\Omega ; A)}\|u\|_{L^{2}(\Omega)}+\|c\|_{L^{\infty}\left(Q_{T}\right)}\|u\|_{L^{2}(\Omega)}^{2} .
\end{aligned}
$$

Using Cauchy's inequality with $\varepsilon=\mu_{1} /\left[2||[\sqrt{M}]^{-1} b \|_{L^{\infty}\left(Q_{T}\right)}\right]$ on the second term above yields (5.38) with

$$
\beta=\frac{\mu_{1}}{2} \quad \text { and } \quad \gamma=\frac{\mu_{2}^{2}\|\|[\sqrt{M}]^{-1} b \mid \|_{L^{\infty}\left(Q_{T}\right)}^{2}}{2 \mu_{1}}+\|c\|_{L^{\infty}\left(Q_{T}\right)} .
$$

This completes the proof.

As in the degenerate elliptic case, one has weak comparison and maximum principles compatible with the existence theory established above for degenerate parabolic equations with the regularity and structural conditions that we have imposed. Moreover, their proofs involve simple adaptations of the arguments used in the non degenerate setting and hence we will merely state the results, which rely on the notion of weak subsolutions/supersolutions.

Definition 5.11 Let $A$ and $\Omega$ satisfy (2.1) and (2.3) and let $f \in L^{2}\left(0, T ; H^{-1}(\Omega ; A)\right)$.

(a) We say that $u \in L^{2}\left(0, T ; H^{1}(\Omega ; A)\right)$ with $\frac{d u}{d t} \in L^{2}\left(0, T ; H^{-1}(\Omega ; A)\right)$ is a weak subsolution to the equation $\partial_{t} u-L u=f$ in $Q_{T}$ if, for a.e. $t \in(0, T)$,

$$
\left\langle\frac{d u}{d t}(t), v\right\rangle+\mathcal{B}(t ; u, v) \leq\langle f(t) v\rangle \quad \text { for any } v \in C_{0}^{1}(\Omega), v \geq 0
$$

(b) We say that $u \in L^{2}\left(0, T ; H^{1}(\Omega ; A)\right)$ with $\frac{d u}{d t} \in L^{2}\left(0, T ; H^{-1}(\Omega ; A)\right)$ is a weak supersolution to the equation $\partial_{t} u-L u=f$ in $Q_{T}$ if, for a.e. $t \in(0, T)$,

$$
\left\langle\frac{d u}{d t}(t), v\right\rangle+\mathcal{B}(t ; u, v) \geq\langle f(t) v\rangle \quad \text { for any } v \in C_{0}^{1}(\Omega), v \geq 0
$$

Making use of considerations like those recorded in Remark 5.9, one can replace the test function space $C_{0}^{1}(\Omega)$ with $H_{0}^{1}(\Omega ; A)$ in the above definition. The weak comparison principle is the following result.

Theorem 5.12 Let the assumptions of Theorem 5.10 be satisfied. Let $u \in L^{2}\left(0, T ; H^{1, A}(\Omega)\right)$ with $\frac{d u}{d t} \in L^{2}\left(0, T ; H^{-1}(\Omega ; A)\right)$ be a subsolution to the equation $\partial_{t} u-L u=0$ in $Q_{T}$ such that $u \leq 0$ on $\partial \Omega \times(0, T)$ and $u(x, 0) \leq 0$ for a.e. $x \in \Omega$. Then

$$
u \leq 0 \text { a.e. in } Q_{T} \text {. }
$$

A direct consequence of Theorem 5.12 is the following maximum principle.

Corollary 5.13 Let assumptions of Theorem 5.10 be satisfied. Let $u$ be a weak solution to problem (5.29) with $f=0$ in the sense of distributions. Suppose that $u_{0} \in L^{\infty}(\Omega)$. Then $u \in L^{\infty}\left(Q_{T}\right)$ and

$$
\|u\|_{L^{\infty}\left(Q_{T}\right)} \leq\left\|u_{0}\right\|_{L^{\infty}(\Omega)} .
$$




\subsection{Degenerate hyperbolic problems}

Next we consider the Cauchy-Dirichlet problem associated to equation (1.5); that is

$$
\begin{cases}\partial_{t}^{2} u=L u+f(x, t) & \text { in } Q_{T}:=\Omega \times(0, T] \\ u=0 & \text { in } \partial \Omega \times(0, T], \\ \partial_{t} u=v_{0} & \text { in } \Omega \times\{0\} \\ u=u_{0} & \text { in } \Omega \times\{0\}\end{cases}
$$

where $L$ has the form $L u:=\operatorname{div}\{M(x, t) \nabla u\}+\langle b(x, t), \nabla u\rangle+c(x, t) u, f \in L^{2}\left(0, T ; L^{2}(\Omega)\right)$, $u_{0} \in H_{0}^{1}(\Omega ; A)$ and $v_{0} \in L^{2}(\Omega)$. Under suitable assumptions on the coefficients $(M, b, c)$ the following notion of weak solution makes sense.

Definition 5.14 Given $f \in L^{2}\left(0, T ; L^{2}(\Omega)\right), u_{0} \in H_{0}^{1}(\Omega ; A)$ and $v_{0} \in L^{2}(\Omega)$. We say that $u$ is a weak solution of (5.42) provided:

$$
\begin{gathered}
u \in L^{2}\left(0, T ; H_{0}^{1}(\Omega ; A)\right), \quad \frac{d u}{d t} \in L^{2}\left(0, T ; L^{2}(\Omega)\right), \quad \text { and } \quad \frac{d^{2} u}{d t^{2}} \in L^{2}\left(0, T ; H^{-1}(\Omega: A)\right) ; \\
\frac{d^{2}}{d t^{2}}\langle u(\cdot), v\rangle+\mathcal{B}(\cdot ; u(\cdot), v)=\langle f(\cdot), v\rangle \quad \text { in } \quad \mathcal{D}^{\prime}((0, T)), \forall v \in H_{0}^{1}(\Omega ; A) ;
\end{gathered}
$$

and

$$
u(0)=u_{0}, \frac{d u}{d t}(0)=v_{0}
$$

where

$$
\mathcal{B}(t, u, v):=\int_{\Omega}\{\langle M(x, t) \nabla u, \nabla v\rangle-\langle b(x, t), \nabla u\rangle v-c(x, t) u v\} d x \quad u, v \in H_{0}^{1}(\Omega ; A) .
$$

is the natural family of $t$-dependent bilinear forms and $\nabla u, \nabla v$ are the quasi-gradients of $u, v$.

As in the parabolic case, some remarks concerning the definition are in order.

Remark 5.15 Given u satisfying condition (5.43) in Definition 5.14.

(a) $u$ admits a representative in $C\left([0, T] ; H_{0}^{1}(\Omega ; A)\right)$ with $\frac{d u}{d t} \in C\left([0, T] ; L^{2}(\Omega)\right)$ (see Remark 1 in section 18.5 of [6]). Hence the initial condition (5.45) makes sense.

(b) Ensuring that $\mathcal{B}(t, \cdot, \cdot))$ is a bounded bilinear form for each fixed $t$, one need only check that (5.44) holds for each $v \in C_{0}^{1}(\Omega)$. which is dense in $H_{0}^{1}(\Omega ; A)$.

(c) Ensuring that the map $t \in[0, T] \rightarrow \mathcal{B}(t, \cdot, \cdot))$ is continuous, then the condition (5.44) is equivalent to

$$
\left\langle\frac{d^{2} u}{d t^{2}}(t), v\right\rangle+\mathcal{B}(t, u(t), v)=\langle f(t), v\rangle \text { for a.e. } t \in(0, T), \text { for every } v \in C_{0}^{1}(\Omega) .
$$

In order to treat the initial value problem (5.45) for $u(\cdot)$ satisfying the second order evolution equation (5.44), we will make use of a special case of Theorem 1 of [6] which states that a unique solution in the sense of Definition 5.14 exists, provided that the family of bilinear forms (5.46) can be split as

$$
\mathcal{B}(t, \cdot, \cdot)=\mathcal{B}_{0}(t, \cdot, \cdot)+\mathcal{B}_{1}(t, \cdot, \cdot), \quad t \in[0, T]
$$

where

$$
\mathcal{B}_{k}(t, \cdot, \cdot) \text { is bounded on } H_{0}^{1}(\Omega ; A) \times H_{0}^{1}(\Omega ; A), \quad \forall t \in[0, T], k=0,1 \text {. }
$$


The principal part $\mathcal{B}_{0}$ is assumed to satisfy the following three properties:

$$
\begin{gathered}
\forall u, v \in H_{0}^{1}(\Omega ; A), \quad t \mapsto \mathcal{B}_{0}(t, u, v) \text { defines a } C^{1} \text { function on }[0, T]: \\
\forall u, v \in H_{0}^{1}(\Omega ; A), \quad \mathcal{B}_{0}(t, u, v)=\mathcal{B}_{0}(t, v, u) \quad \forall t \in[0, T] ;
\end{gathered}
$$

and there exist positive constants $\beta, \gamma$ such that

$$
\left|\mathcal{B}_{0}(t, u, u)\right| \geq \beta\|u\|_{H_{0}^{1}(\Omega ; A)}^{2}-\gamma\|u\|_{L^{2}(\Omega)}^{2}, \forall t \in[0, T], \forall u \in H_{0}^{1}(\Omega ; A) .
$$

The lower order part $\mathcal{B}_{1}$ is assumed to satisfy the following two properties

$$
\forall u, v \in H_{0}^{1}(\Omega ; A), \quad t \mapsto \mathcal{B}_{1}(t, u, v) \text { defines a continuous function on }[0, T]:
$$

and there exists a positive constant $\alpha_{1}$

$$
\left|\mathcal{B}_{1}(t, u, v)\right| \leq \alpha_{1}|| u\left\|_{H_{0}^{1}(\Omega ; A)}|| v\right\|_{L^{2}(\Omega)}, \forall t \in[0, T], \forall u, v \in H_{0}^{1}(\Omega ; A) .
$$

Before stating the application of this abstract result, we wish to make a few remarks about the hypotheses. Using the $L^{2}$-norm on $v$ in (5.54) is what makes $\mathcal{B}_{1}$ a term of lower order. Combining the pointwise boundedness (5.49) of $\mathcal{B}_{0}$ with the continuity of the map in (5.50) yields the uniform bound

$$
\left|\mathcal{B}_{0}(t, u, v)\right| \leq \alpha_{0}\|u\|_{H_{0}^{1}(\Omega ; A)}\|v\|_{H_{0}^{1}(\Omega ; A)}, \forall t \in[0, T], \forall u, v \in H_{0}^{1}(\Omega ; A),
$$

for some constant $\alpha_{0}>0$. This uniform boundedness property follows from the Baire category theorem in the same way as one proves the uniform boundedness principle for a pointwise bounded family of linear functionals. Moreover, the derivative with respect to $t$ of the map in (5.50) will take values in the space of bounded symmetric bilinear forms and the continuity of the map $t \mapsto \frac{d}{d t} \mathcal{B}_{0}(t, u, v)$ yields an estimate like (5.55) for $\frac{d}{d t} \mathcal{B}_{0}(t, u, v)$. Control on this family of maps is needed in the a priori estimates required for passing to the limit in the Galerkin approximation scheme.

The needed regularity, boundedness and coercivity will follow by making suitable hypotheses on the coefficients $(M, b, c)$ of the degenerate elliptic operator $L$. For the statement, we denote by $C_{x, t}^{0,1}\left(\overline{Q_{T}}, \operatorname{Sym}_{n}^{+}(\mathbb{R})\right)$ the space of continuous functions on $\overline{Q_{T}}$ taking values in symmetric nonnegative definite matrices whose first order spatial derivatives are continuous.

Theorem 5.16 Let $A$ and $\Omega$ satisfy the regularity and structural conditions (2.1) and (2.3). Assume that the coefficients $(M, b, c)$ of $L$ satisfy

$$
\begin{gathered}
M \in C_{x, t}^{0,1}\left(\overline{Q_{T}}, \operatorname{Sym}_{n}^{+}(\mathbb{R})\right), \\
{[\sqrt{M}]^{-1} b \in C\left(\overline{Q_{T}}, \mathbb{R}^{n}\right) \quad \text { and } \quad c \in C\left(\overline{Q_{T}}, \mathbb{R}\right)}
\end{gathered}
$$

and there exist positive constants $\mu_{1}, \mu_{2}$ such that

$$
\mu_{1} A(x) \leq M(x, t) \leq \mu_{2} A(x), \quad \forall t \in(0, T), \forall x \in \Omega,
$$

with the inequalities in the sense of quadratic forms. Then there exists a unique weak solution to the Cauchy-Dirichlet problem (5.42) in the sense of Definition 5.14.

Proof. We split $\mathcal{B}$ in the obvious way by setting

$$
\mathcal{B}_{0}(t, u, v)=\int_{\Omega}\langle M(x, t) \nabla u, \nabla v\rangle d x \quad \text { and } \quad \mathcal{B}_{1}(t, u, v)=\int_{\Omega}\{\langle b(x, t), \nabla u\rangle v+c(x, t) u v\} d x .
$$

The boundedness properties (5.49), (5.54) and the weak coercivity (5.52) follow from the proof of Theorem 5.10 where the same properties were shown under weaker assumptions. The regularity properties (5.50) and (5.53) are easily verified with the aid of Theorem 4.3. 


\section{Examples of matrix valued weights}

In this section, we will give a few simple examples to illustrate the structural hypotheses made on the matrix $A$ and its singular set $\mathcal{S}$ in order to ensure the validity of the Poincaré inequality of Theorem 3.1 for each $p \in[1, \infty)$. The first example shows that there exist matrix valued weights $A$ which satisfy (2.1) and (2.3), but which do not admit a uniformly elliptic direction, as required in [20].

Example 6.1 Let $\Omega:=\left\{(x, y, z) \in \mathbb{R}^{3}: x^{2}+y^{2}<4,|z|<1\right\}$ and let $\alpha>1$ be arbitrary. Define the matrix $A$ for $(x, y, z)$ in a $C^{1,1}$ neighborhood $\widetilde{\Omega}$ of $\bar{\Omega}$ by

$$
A(x, y, z):=\left(\begin{array}{ccc}
x^{2} & x y & 0 \\
x y & y^{2} & 0 \\
0 & 0 & 0
\end{array}\right)+\left(\left(x^{2}+y^{2}-1\right)^{2}+z^{2}\right)^{\alpha}\left(\begin{array}{ccc}
1 & 0 & 0 \\
0 & 1 & 0 \\
0 & 0 & 1
\end{array}\right) .
$$

The matrix valued function $A$ satisfies the condition (2.1) on $\widetilde{\Omega}$. Indeed, $\alpha>1$ ensures the required regularity of parts $(i)$ and $(i i)$ and the nonnegativity follows from the fact that $A$ is the sum of nonnegative definite matrices. Denoting by $\psi:=\left(\left(x^{2}+y^{2}-1\right)^{2}+z^{2}\right)^{\alpha} \geq 0$, one finds that $\operatorname{det} A=\psi^{2}\left(\psi+x^{2}+y^{2}\right)$ and hence the degeneracy set is the zero locus of $\psi$; that is,

$$
\mathcal{S}=\left\{(x, y, z) \in \mathbb{R}^{3}: x^{2}+y^{2}=1, z=0\right\},
$$

which is a compact connected $C^{\infty}$ submanifold without boundary of codimension 2 contained in $\Omega$. In order to have condition (2.3), it remains to check the orthogonal rank condition (2.3)-(iii). For any $\left(x_{0}, y_{0}, 0\right) \in \mathcal{S}$ we have

$$
\operatorname{Ker}\left(A\left(x_{0}, y_{0}, 0\right)\right)=\operatorname{Span}\left\langle(0,0,1),\left(y_{0},-x_{0}, 0\right)\right\rangle \quad \text { and } \quad T_{\left(x_{0}, y_{0}, 0\right)} \mathcal{S}=\operatorname{Span}\left\langle\left(y_{0},-x_{0}, 0\right)\right\rangle .
$$

Thus

$$
N_{\left(x_{0}, y_{0}, 0\right)} \mathcal{S}=\left(T_{\left(x_{0}, y_{0}, 0\right)} \mathcal{S}\right)^{\perp}=\operatorname{Span}\left\langle(0,0,1),\left(x_{0}, y_{0}, 0\right)\right\rangle
$$

and

$$
\left(x_{0}, y_{0}, 0\right) \in N_{\left(x_{0}, y_{0}, 0\right)} \mathcal{S} \backslash \operatorname{Ker}\left(A\left(x_{0}, y_{0}, 0\right)\right) .
$$

In particular, the hypotheses (2.1) and (2.3) are satisfied on $\widetilde{\Omega}$ and Theorem 3.1 applies. Then Corollary 3.2 yields the validity of the Poincaré inequality on $\Omega$.

On the other hand, $A$ does not admit any uniformly elliptic direction, which means that there is no vector $\xi$ of norm one such that there exists $\beta>0$ such that

$$
\langle A(x, y, z) \xi, \xi\rangle \geq \beta>0, \quad \forall(x, y, z) \in \Omega,
$$

as it is required in [20]. In fact, for every $\xi \equiv\left(\xi_{1}, \xi_{2}, \xi_{3}\right) \in \mathbb{R}^{3} \backslash\{0\}$ there exists a point $\left(x_{0}, y_{0}, 0\right) \in \mathcal{S}$ such that $\xi \in \operatorname{Ker}\left(A\left(x_{0}, y_{0}, 0\right)\right)$. Indeed, if $\xi_{1}^{2}+\xi_{2}^{2} \neq 0$, then one can choose

$$
\left(x_{0}, y_{0}, 0\right):=\left(\frac{\xi_{2}}{\sqrt{\xi_{1}^{2}+\xi_{2}^{2}}},-\frac{\xi_{1}}{\sqrt{\xi_{1}^{2}+\xi_{2}^{2}}}, 0\right) \in \mathcal{S},
$$

while if $\xi_{1}=\xi_{2}=0$, then one can choose any $\left(x_{0}, y_{0}, 0\right) \in \mathcal{S}$.

We next give examples which show that the Poincaré inequality can fail if the orthogonal rank condition (2.3)-(iii) does not hold. In all of the examples we present, $\operatorname{Ker}(A(x))=N_{x} \mathcal{S}$ for each $x \in \mathcal{S}$ although it would suffice to show the existence of $x_{0} \in \mathcal{S}$ for which $N_{x_{0}} \mathcal{S} \subset$ $\operatorname{Ker}\left(A\left(x_{0}\right)\right)$. The first counterexample has $\mathcal{S}$ of codimension 2 and $A$ not totally degenerate on $\mathcal{S}$. 
Example 6.2 Let $\Omega:=\left\{(x, y, z) \in \mathbb{R}^{3}: x^{2}+y^{2}<4,|z|<1\right\}$ and let $\alpha>1$ be a parameter to be chosen sufficiently large. Define the matrix $A$ for $(x, y, z)$ in a $C^{1,1}$ neighborhood $\widetilde{\Omega}$ of $\bar{\Omega}$ by

$$
A(x, y, z):=\left(\begin{array}{ccc}
y^{2} & -x y & 0 \\
-x y & x^{2} & 0 \\
0 & 0 & 0
\end{array}\right)+\left(\left(x^{2}+y^{2}-1\right)^{2}+z^{2}\right)^{\alpha}\left(\begin{array}{ccc}
1 & 0 & 0 \\
0 & 1 & 0 \\
0 & 0 & 1
\end{array}\right)
$$

As in Example 6.1, The matrix valued function $A$ satisfies the condition (2.1) on $\widetilde{\Omega}$ and has the same determinant as before and hence the associated degeneracy set is again

$$
\mathcal{S}=\left\{(x, y, z) \in \mathbb{R}^{3}: x^{2}+y^{2}=1, z=0\right\} .
$$

Hence the validity of condition (2.3) reduces to the orthogonal rank condition (2.3)-(iii). For any $\left(x_{0}, y_{0}, 0\right) \in \mathcal{S}$ we have

$$
\operatorname{Ker}\left(A\left(x_{0}, y_{0}, 0\right)\right)=\operatorname{Span}\left\langle(0,0,1),\left(x_{0}, y_{0}, 0\right)\right\rangle
$$

and

$$
T_{\left(x_{0}, y_{0}, 0\right)} \mathcal{S}=\operatorname{Span}\left\langle\left(y_{0},-x_{0}, 0\right)\right\rangle
$$

Thus,

$$
N_{\left(x_{0}, y_{0}, 0\right)} \mathcal{S}=\left(T_{\left(x_{0}, y_{0}, 0\right)} \mathcal{S}\right)^{\perp}=\operatorname{Span}\left\langle(0,0,1),\left(x_{0}, y_{0}, 0\right)\right\rangle=\operatorname{Ker}\left(A\left(x_{0}, y_{0}, 0\right)\right)
$$

and the condition (2.3)-(iii) fails to hold and we cannot affirm the validity of the Poincaré inequality.

In fact, the Poincaré inequality does not hold. To show this, it suffices to produce a sequence of functions $\left\{v_{n}\right\}_{n \in \mathbb{N}} \subset C_{0}^{\infty}(\Omega)$ such that

$$
\left\|v_{n}\right\|_{p} \rightarrow \infty \quad \text { as } n \rightarrow \infty, \quad \int_{\Omega}\left\langle A \nabla v_{n}, \nabla v_{n}\right\rangle^{\frac{p}{2}} d x d y d z \leq M \quad \text { for every } n \in \mathbb{N}
$$

for some $M>0$. An example of such a sequence can be constructed in the following way. Let $\varphi \in C_{0}^{\infty}(\Omega)$ be a function satisfying $0 \leq \varphi \leq 1$ and $\varphi \equiv 1$ on $\mathcal{U}:=\left\{x^{2}+y^{2} \leq 9 / 4,|z|<1 / 2\right\}$ which contains $\mathcal{S}$ and satisfies $\overline{\mathcal{U}} \subset \Omega$. Define the sequence of $C_{0}^{\infty}(\Omega)$ functions

$$
v_{n}(x, y, z):=\varphi(x, y, z)\left(\left(x^{2}+y^{2}-1\right)^{2}+z^{2}+\frac{1}{n}\right)^{-\beta} \quad \text { for all }(x, y, z) \in \Omega,
$$

where $\beta$ is a positive constant such that $\beta>\frac{3}{2 p}$. Using the monotone convergence theorem, it is easy to see that

$$
\lim _{n \rightarrow \infty} \int_{\Omega} v_{n}^{p} d x d y d z=\infty .
$$

On the other hand, if one sets $\psi_{n}:=\left(x^{2}+y^{2}-1\right)^{2}+z^{2}+\frac{1}{n}$ then

$$
\nabla v_{n}(x, y, z)=\psi_{n}^{-\beta} \nabla \varphi(x, y, z)-\beta \varphi \psi_{n}^{-\beta-1} \nabla \psi_{n}(x, y, z):=V_{n}+W_{n} .
$$

For each $(x, y, z) \in \Omega$ one has

$$
\nabla \psi_{n}(x, y, z)=\left(4 x\left(x^{2}+y^{2}-1\right), 4 y\left(x^{2}+y^{2}-1\right), 2 z\right) \in \operatorname{Ker}(\widetilde{A}(x, y, z))
$$

where

$$
\tilde{A}(x, y, z):=\left(\begin{array}{ccc}
y^{2} & -x y & 0 \\
-x y & x^{2} & 0 \\
0 & 0 & 0
\end{array}\right)
$$


Making use of the decomposition (6.62), since $A \geq 0$ and is self-adjoint, elementary inequalities yield the following pointwise bound on $\Omega$ :

$$
\left\langle A \nabla v_{n}, \nabla v_{n}\right\rangle^{\frac{p}{2}} \leq 2^{p-\frac{1}{2}}\left(\left|\sqrt{A} V_{n}\right|^{p}+\left|\sqrt{A} W_{n}\right|^{p}\right) .
$$

Notice that $A=\tilde{A}+\psi^{\alpha} I$ if one defines $\psi(x, y, z):=\left(x^{2}+y^{2}-1\right)^{2}+z^{2}$. Using (6.63), $\operatorname{supp}\left(V_{n}\right) \subset \Omega \backslash \mathcal{U}$, the lower bound $\psi_{n}>\psi$ and the upper bound $\varphi \leq 1$, one integrates (6.64) to find $C>0$ such that

$$
\int_{\Omega}\left\langle A \nabla v_{n}, \nabla v_{n}\right\rangle^{\frac{p}{2}} d x d y d z \leq C\left(\int_{\Omega \backslash \mathcal{U}}|\sqrt{A} \nabla \varphi|^{p} \psi^{-p \beta} d x d y d z+\int_{\Omega} \psi^{-p \beta-\frac{p}{2}+\frac{p \alpha}{2}} d x d y d z\right),
$$

for each $n \in \mathbb{N}$. This expression is bounded by some positive constant $M$, provided that $\alpha \geq 2 \beta+1$. Combining this with (6.61) yields (6.60).

The following counterexample again has $\mathcal{S}$ of codimension 2, but $A$ is totally degenerate on $\mathcal{S}$, which is a single point.

Example 6.3 Let $\Omega:=\left\{(x, y) \in \mathbb{R}^{2}: x^{2}+y^{2}<1\right\}$ and let $\alpha>1$ be a parameter to be chosen sufficiently large. Define the weight $A$ for $(x, y) \in \bar{\Omega}$ by

$$
A(x, y):=\left(\begin{array}{cc}
\left(x^{2}+2 y^{2}\right)^{\alpha} & 0 \\
0 & \left(2 x^{2}+y^{2}\right)^{\alpha}
\end{array}\right) .
$$

Since $\alpha>1$ and $A$ is nonnegative definite, one has (2.1). Clearly $\mathcal{S}=\{(0,0)\}$ and since $A$ vanishes there, the condition (2.3)-(iii) is not satisfied.

The Poincarè inequality fails as again one can construct $\left\{v_{n}\right\}_{n \in \mathbb{N}} \subset C_{0}^{\infty}(\Omega)$ satisfying (6.60). Indeed, let $\varphi \in C_{0}^{\infty}(\Omega)$ satisfy $0 \leq \varphi \leq 1$ and $\varphi(x, y)=1$ for every $(x, y) \in \mathcal{U}:=$ $\left\{(x, y) \in \mathbb{R}^{2}: x^{2}+y^{2} \leq \frac{1}{2}\right\}$ and define

$$
v_{n}(x, y)=\varphi(x, y)\left(x^{2}+y^{2}+\frac{1}{n}\right)^{-\beta} .
$$

Arguing as in Example 6.2, one finds that (6.60) holds for $\beta>\frac{1}{p}$ and $\alpha \geq 2 \beta+1$.

We conclude with two examples which relax the condition $(2.3)-(i i)$ to allow $\operatorname{codim}(\mathcal{S})=1$ but for which the topological condition (3.2) holds. As explained in Remark 3.5, one will have the Poincarè inequality of Theorem 3.1 provided that the remainder of the conditions (2.1) and (2.3) hold. The following illustrates the failure of the Poincarè inequality in the absence of the orthogonal rank condition (2.3)-(iii).

Example 6.4 Let $\Omega:=\left\{(x, y) \in \mathbb{R}^{2}: \frac{1}{2}<x^{2}+y^{2}<2\right\}$ and let $\alpha>1 / 2$ be a parameter to be chosen sufficiently large. Define the matrix A for $(x, y) \in \bar{\Omega}$ by

$$
A(x, y):=\left(\begin{array}{cc}
y^{2} & -x y \\
-x y & x^{2}
\end{array}\right)+\left(x^{2}+y^{2}-1\right)^{2 \alpha}\left(\begin{array}{ll}
1 & 0 \\
0 & 1
\end{array}\right) .
$$

Since $\alpha>1 / 2$ and $A$ is the sum of two nonnegative matrix valued functions, one has (2.1). Denoting by $\psi:=\left(x^{2}+y^{2}-1\right)^{2 \alpha} \geq 0$, one has $\operatorname{det} A=\psi\left(\psi+x^{2}+y^{2}\right)$ and hence

$$
\mathcal{S}=\left\{(x, y) \in \mathbb{R}^{2}: x^{2}+y^{2}=1\right\}
$$

which is a compact connected smooth manifold contained in $\Omega$ of codimension 1. Since

$$
\Omega \backslash \mathcal{S}=\left\{(x, y) \in \mathbb{R}^{2}: \frac{1}{2}<x^{2}+y^{2}<1\right\} \cup\left\{(x, y) \in \mathbb{R}^{2}: 1<x^{2}+y^{2}<2\right\}:=\Omega_{1} \cup \Omega_{2},
$$


the open connected components $\Omega_{1}, \Omega_{2}$ satisfy the condition (3.2); that is, $\partial \Omega_{i} \cap \partial \Omega \neq \emptyset$ for $i=1,2$, However, for every $(x, y) \in \mathcal{S}$ one has

$$
T_{(x, y)} \mathcal{S}=\operatorname{Span}\langle(y,-x)\rangle \quad \text { and } \quad N_{(x, y)} \mathcal{S}=\operatorname{Span}\langle(x, y)\rangle=\operatorname{Ker}(A(x, y))
$$

and hence the condition (2.3)-(iii) is not satisfied.

The Poincarè inequality fails as a sequence $\left\{v_{n}\right\}_{n \in \mathbb{N}} \subset C_{0}^{\infty}(\Omega)$ satisfying (6.60) is easily constructed. Indeed, pick $\varphi \in C_{0}^{\infty}(\Omega)$ with $\varphi \equiv 1$ for $3 / 4 \leq x^{2}+y^{2} \leq 9 / 4$ and set

$$
v_{n}(x, y)=\left(\left(x^{2}+y^{2}-1\right)^{2}+\frac{1}{n}\right)^{-\beta} \varphi(x, y) .
$$

The same reasoning as the previous examples yields (6.60) for $\beta>\frac{1}{2 p}$ and $\alpha \geq 4+4 \beta$.

Next we give an example of $\mathcal{S}$ having codimension 1 which corrects the defect in the orthogonal rank condition of the previous example.

Example 6.5 Let $\Omega:=\left\{(x, y) \in \mathbb{R}^{2}: \frac{1}{2}<x^{2}+y^{2}<2\right\}$ and let $\alpha>\frac{1}{2}$ be arbitrary. For every $(x, y) \in \bar{\Omega}$ define

$$
A(x, y)=\left(\begin{array}{ll}
x^{2} & x y \\
x y & y^{2}
\end{array}\right)+\left(x^{2}+y^{2}-1\right)^{2 \alpha}\left(\begin{array}{ll}
1 & 0 \\
0 & 1
\end{array}\right) .
$$

With respect to Example 6.4, changing signs on the off-diagonal entries leaves all of the relevant properties of $A$ and $\mathcal{S}$ unchanged with the exception of $\operatorname{Ker} A$, which is now

$$
\operatorname{Ker}(A(x, y))=T_{(x, y)} \mathcal{S}=\operatorname{Span}\langle(y,-x)\rangle
$$

so that for each $(x, y) \in \mathcal{S}=\left\{(x, y) \in \mathbb{R}^{2}: x^{2}+y^{2}=1\right\}$ one has that the rank of $A(x, y)$ restricted to $N_{(x, y)} \mathcal{S}$ is one and the condition (2.3)-(iii) is satisfied. Thus the Poincaré inequality (3.1) holds in accordance with Remark 3.5.

Finally, we give an example which shows that Sobolev inequalities with a strict gain in summability may fail for matrices $A$ which support the inequality (1.2).

Example 6.6 Let $\Omega:=\left[-\frac{1}{2} e^{-2}, \frac{1}{2} e^{-2}\right] \times\left[-\frac{1}{2}, \frac{1}{2}\right], p \geq 1$ and let $\alpha \geq 2+\frac{2}{p}$ be arbitrary. For every $(x, y) \in \bar{\Omega}$ define

$$
A(x, y)=\left(\begin{array}{cc}
|x|^{\alpha} & 0 \\
0 & 1
\end{array}\right)
$$

Then the coefficients of the matrix $A$ are $C^{1}$, the degeneracy set is $\mathcal{S}=\left\{(0, y) \mid y \in\left[-\frac{1}{2}, \frac{1}{2}\right]\right\}$ and $(0,1)$ is a direction of uniform ellipticity for the matrix $A$. By the results in [20] one has the validity of a Poincare inequality of the form (3.1) for every $u \in C_{0}^{1}(\Omega)$. In particular, the results of Sections 4 and 5 of the present work apply to the matrix $A$.

Let $\psi \in C_{0}^{\infty}(\Omega)$ be a smooth cutoff function such that $0 \leq \psi \leq 1$ in $\Omega$ and $\psi \equiv 1$ in $\Theta:=\left[-\frac{1}{4} e^{-2}, \frac{1}{4} e^{-2}\right] \times\left[-\frac{1}{4}, \frac{1}{4}\right]$. Define on $\Omega$

$$
\varphi_{n}(x, y)=\left[\left(x^{2}+\frac{1}{n}\right)^{\frac{1}{2}}\left(\log \left(x^{2}+\frac{1}{n}\right)\right)^{2}\right]^{-\frac{1}{p}} \in C^{\infty}(\Omega)
$$

and $u_{n}(x, y)=\psi(x, y) \varphi_{n}(x, y)$. Then one has

$$
\begin{aligned}
\frac{\partial}{\partial x} \varphi_{n}(x, y)= & -\frac{1}{p}\left[\left(x^{2}+\frac{1}{n}\right)^{\frac{1}{2}}\left(\log \left(x^{2}+\frac{1}{n}\right)\right)^{2}\right]^{-\frac{1+p}{p}} \times \\
& {\left[x\left(x^{2}+\frac{1}{n}\right)^{-\frac{1}{2}}\left(\log \left(x^{2}+\frac{1}{n}\right)\right)^{2}+4 x \log \left(x^{2}+\frac{1}{n}\right)\left(x^{2}+\frac{1}{n}\right)^{-\frac{1}{2}}\right] }
\end{aligned}
$$


so that

$$
\begin{aligned}
& \left|\sqrt{A} \nabla \varphi_{n}\right|^{2} \\
& \quad=\left|\left\langle A \nabla \varphi_{n}, \nabla \varphi_{n}\right\rangle\right|=|x|^{\alpha}\left(\frac{\partial \varphi_{n}}{\partial x}\right)^{2} \\
& \quad \leq C|x|^{\alpha}\left[\left(x^{2}+\frac{1}{n}\right)\left(\log \left(x^{2}+\frac{1}{n}\right)\right)^{4}\right]^{-\frac{1+p}{p}}\left[\left(\log \left(x^{2}+\frac{1}{n}\right)\right)^{4}+\left(\log \left(x^{2}+\frac{1}{n}\right)\right)^{2}\right] \\
& \quad=C|x|^{\alpha}\left[\left(x^{2}+\frac{1}{n}\right)^{-1-\frac{1}{p}}\left(\log \left(x^{2}+\frac{1}{n}\right)\right)^{-\frac{4}{p}}+\left(x^{2}+\frac{1}{n}\right)^{-1-\frac{1}{p}}\left(\log \left(x^{2}+\frac{1}{n}\right)\right)^{-\frac{4}{p}-2}\right] .
\end{aligned}
$$

Now note that the function $t \mapsto t^{a}|\log t|^{b}$, for $a, b<0$, is decreasing in $\left[0, e^{-\frac{b}{a}}\right]$. Choosing $a=-1-\frac{1}{p}, b=-\frac{4}{p}$ we obtain $e^{-\frac{b}{a}}=e^{-\frac{4}{p+1}} \geq e^{-2}$, while choosing $a=-1-\frac{1}{p}, b=-\frac{4}{p}-2$ we obtain $e^{-\frac{b}{a}}=e^{-\frac{4+2 p}{p+1}} \geq e^{-3}$. Then, for $n \in \mathbb{N}$ sufficiently large, we have $x^{2}<x^{2}+\frac{1}{n}<e^{-3}$ for every $(x, y) \in \Omega$. Thus we deduce

$$
\left|\sqrt{A} \nabla \varphi_{n}\right|^{2} \leq C|x|^{\alpha}\left[x^{-2-\frac{2}{p}}(\log |x|)^{-\frac{4}{p}}+x^{-2-\frac{2}{p}}(\log |x|)^{-\frac{4}{p}-2}\right] \leq C,
$$

for $\alpha \geq 2+\frac{2}{p}$, independently of $n \in \mathbb{N}$. Therefore, also recalling the properties of $\psi$, for sufficiently large $n \in \mathbb{N}$ we have

$$
\begin{aligned}
\left\langle A \nabla u_{n}, \nabla u_{n}\right\rangle^{\frac{p}{2}} & =\left|\sqrt{A} \nabla u_{n}\right|^{p} \leq\left(\left|\psi \sqrt{A} \nabla \varphi_{n}\right|+\left|\varphi_{n} \sqrt{A} \nabla \psi\right|\right)^{p} \\
& \leq C\left(\left|\psi \sqrt{A} \nabla \varphi_{n}\right|^{p}+\left|\varphi_{n} \sqrt{A} \nabla \psi\right|^{p}\right) \\
& \leq C\left(1+\left(x^{2}+\frac{1}{n}\right)^{-\frac{1}{2}}\left(\log \left(x^{2}+\frac{1}{n}\right)\right)^{-2}\right) \\
& \leq C\left(1+|x|^{-1}(\log |x|)^{-2}\right)
\end{aligned}
$$

in $\Omega$, independently of $n \in \mathbb{N}$. Thus we conclude that $u_{n} \in W_{0}^{1, p}(\Omega ; A)$ and that there exists a constant $M>0$ such that $\left\|u_{n}\right\|_{W_{0}^{1, p}(\Omega ; A)} \leq M$ for every $n \in \mathbb{N}$. By the Poincaré inequality, the sequence $u_{n}$ is bounded also in $L^{p}(\Omega)$.

Now we show that a Sobolev inequality with strict gain, of the form: for some $\varepsilon>0$ there exists a constant $C>0$ such that

$$
\|u\|_{L^{p(1+\epsilon)}(\Omega)} \leq C\left(\int_{\Omega}\langle A(x, y) \nabla u, \nabla u\rangle^{\frac{p}{2}} d x d y\right)^{\frac{1}{p}}, \forall u \in C_{0}^{1}(\Omega),
$$

does not hold in this setting. Indeed, we claim that for the sequence $u_{n}$ defined above one has

$$
\lim _{n \rightarrow+\infty}\left\|u_{n}\right\|_{L^{p(1+\epsilon)}(\Omega)}=+\infty, \quad \forall \varepsilon>0 .
$$

To show the claim, we estimate

$$
\begin{aligned}
\int_{\Omega}\left|u_{n}(x, y)\right|^{p(1+\epsilon)} d x d y & \geq \int_{-\frac{1}{4}}^{\frac{1}{4}} \int_{-\frac{1}{4} e^{-2}}^{\frac{1}{4} e^{-2}}\left|\varphi_{n}(x, y)\right|^{p(1+\epsilon)} d x d y \\
& =\int_{0}^{\frac{1}{4} e^{-2}}\left(x^{2}+\frac{1}{n}\right)^{-\frac{1+\epsilon}{2}}\left(\log \left(x^{2}+\frac{1}{n}\right)\right)^{-2(1+\epsilon)} d x \\
& \rightarrow \frac{1}{4^{1+\epsilon}} \int_{0}^{\frac{1}{4} e^{-2}} x^{-(1+\epsilon)}(\log x)^{-2(1+\epsilon)} d x=+\infty
\end{aligned}
$$

by the monotone convergence theorem. Thus (6.66) holds while at the same time the sequence $u_{n}$ is bounded in $W_{0}^{1, p}(\Omega ; A)$, and hence there is no $C>0$ such that (6.65) holds. 


\section{References}

[1] L. Ambrosio, "Lecture Notes on Elliptic Partial Differential Equations", Scuola Normale Superiore di Pisa, online http://cvgmt.sns.it/media/doc/paper/1280/PDEAAA.pdf, 2015.

[2] A. Bonfiglioli, E. Lanconelli and F. Uguzzoni, "Stratified Lie Groups and Potential Theory for their Sub-Laplacians", Springer Monographs in Mathematics, Springer-Verlag, Berlin, 2007.

[3] H. Brezis, "Functional Analysis, Sobolev Spaces and Partial Differential Equations", Universitext, Springer, New York, 2011.

[4] S.-K. Chua, S. Rodney and R.L. Wheeden, A compact embedding theorem for generalized Sobolev spaces, Pacific J. Math 265 (2013), 17-57.

[5] F.M. Chiarenza and R.P. Serapioni, A Harnack inequality for degenerate parabolic equations, Comm. Partial Differential Equations 9 (1984), 719-749.

[6] R. Dautray and J-L. Lions, "Mathematical Analysis and Numerical Methods for Science and Technology: Volume 5, Evolution Problems I", Springer-Verlag, Berlin, 1992. 2011.

[7] E.B. Fabes C.E. Kenig and R.P. Serapioni, The local regularity of solutions to degenerate elliptic equations, Comm. Partial Differential Equations 7 (1982), 77-116.

[8] C. Fefferman and D.H. Phong, Subelliptic eigenvalue problems, in "Conference on harmonic analysis in honor of Antoni Zygmund, Vol. I, II (Chicago, Ill., 1981)", 590-606, Wadsworth Math. Ser., Wadsworth, Belmont, CA, 1983.

[9] G. Fichera, Sulle equazioni differenziali lineari ellittico-paraboliche del secondo ordine, Atti Acad. Naz. Lincei. Mem. Cl. Sci. Fis. Mat. Nat. Sez. 1 (8) 5 (1956), 1-30.

[10] G. Fichera, On a unified theory of boundary value problems for elliptic-parabolic equations of second order, in "Boundary Problems in Differential Equations", pp. 97-120, Univ. of Wisconsin Press, Madison, 1960.

[11] R.L. Foote, Regularity of distance function, Proc. of AMS, 92 (1984), 153-155.

[12] B. Franchi and E. Lanconelli, Une métrique associée à une classe d'opérateurs elliptiques dégénérés (French). Conference on linear partial and pseudodifferential operators (Torino, 1982). Rend. Sem. Mat. Univ. Politec. Torino 1983, Special Issue, (1984), 105-114.

[13] B. Franchi and E. Lanconelli, Une métrique associée à une classe d'opérateurs elliptiques dégénérés (French). Conference on linear partial and pseudodifferential operators (Torino, 1982). Rend. Sem. Mat. Univ. Politec. Torino 1983, Special Issue, (1984), 105-114.

[14] A. Friedman, "Stochastic Differential Equations and Applications I, II", Probability and Mathematical Statistics, Vol. 28, Academic Press, New York, 1975,1976.

[15] D. Gilbarg and N. Trudinger, "Elliptic partial differential equations of second order", Classics in Mathematics, Springer-Verlag, Berlin, 2001.

[16] P. Hajlasz and P. Koskela Sobolev met Poincaré, Mem. Amer. Math. Soc. 145 (2000), $\mathrm{x}+101 \mathrm{p}$.

[17] L. Hörmander, Hypoelliptic second order differential equations, Acta. Math. 119 (1967), 147-171.

[18] E. Lanconelli and and A.E.Kogoj X-elliptic operators and X-control distances. Contributions in honor of the memory of Ennio De Giorgi (Italian), Ricerche Mat. 49 (Suppl.) (2000), 223-243.

[19] J-L. Lions and E. Magenes, "Non-homogeneous Boundary Value Problems and Applications I,II,III", Die Grundlehren der mathematischen Wissenschaften, Band 181, 182, 183, Springer-Verlag, New York-Heidelberg, 1972, 1972, 1973. 
[20] D.D. Monticelli and K.R. Payne, Maximum principles for weak solutions of degenerate elliptic equations with a uniformly direction, J. Differential Equations 247 (2009), 19932026 .

[21] D.D. Monticelli and S. Rodney, Existence and spectral theory for weak solutions of Neumann and Dirichlet problems for linear degenerate elliptic operators with rough coefficients, J. Differential Equations 259 (2012), 143-200.

[22] D.D. Monticelli, S. Rodney and R.L. Wheeden, Boundedness of weak solutions of degenerate quasilinear equations with rough coefficients, J. Diff. Int. Eq. 25 (2012), 143-200.

[23] D.D. Monticelli, S. Rodney and R.L. Wheeden, Harnack's inequality and Hölder continuity for weak solutions of degenerate quasilinear equations with rough coefficients, submitted, arXiv:1411.6976.

[24] M.K.V. Murthy and G. Stampacchia, Boundary value problems for some degenerateelliptic operators, Ann. Mat. Pura Appl. (4) 80 (1968), 1-122.

[25] O.A. Olënik and E.V. Radkevič, "Second Order Equations with Nonnegative Characteristic Form", Translated from the Russian by Paul C. Fife, Plenum Press, New York-London, 1973.

[26] M.A. Pozio, F. Punzo, and A. Tesei, Criteria for well-posedness of degenerate elliptic and parabolic problems, J. Math. Pures Appl., 90 (2008), 353-386.

[27] S. Rodney, Existence of weak solutions of linear subelliptic Dirichlet problems with rough coefficients, Canad. J. Math. 64 (2012), 1394-1414.

[28] S. Salsa, "Partial Differential Equations in Action. From Modelling to Theory", Universitext, Springer-Verlag Italia, Milan, 2008.

[29] G. Stampacchia, Problemi al contorno ellittici, con dati discontinui, dotati di soluzioni hölderiane, Ann. Mat. Pura Appl. (4) 51 (1960), 1-37.

[30] E.T. Sawyer and R.L. Wheeden, Hölder continuity of weak solutions to subelliptic equations with rough coefficients, Memoirs Amer. Math. Soc. 847 (2006).

[31] E.T. Sawyer and R.L. Wheeden, Degenerate Sobolev spaces and regularity of subelliptic equations, Trans. Amer. Math. Soc., 362 (2010), 1869-1906. 